\title{
Évolution des formes de l'exploitation agricole dans la campagne normande (2500-30 avant J.-C.)
}

L'exemple des fouilles préventives de la périphérie sud de Caen Evolution of agricultural exploitation types in Norman country (2500-30 BC) The example of preventive excavations in the southern outskirts of Caen

Benjamin van den Bosche, Vincent Carpentier et Cyril Marcigny

\section{OpenEdition}

Journals

Édition électronique

URL : http://journals.openedition.org/rao/813

DOI : $10.4000 /$ rao. 813

ISBN : 978-2-7535-1609-0

ISSN : 1775-3732

Éditeur

Presses universitaires de Rennes

Édition imprimée

Date de publication : 31 décembre 2009

Pagination : $57-83$

ISBN : 978-2-7535-1086-9

ISSN : 0767-709X

\section{Référence électronique}

Benjamin van den Bosche, Vincent Carpentier et Cyril Marcigny, «Évolution des formes de

l'exploitation agricole dans la campagne normande (2500-30 avant J.-C.) ", Revue archéologique de I'Ouest [En ligne], 26 | 2009, mis en ligne le 31 décembre 2011, consulté le 06 décembre 2020. URL http://journals.openedition.org/rao/813 ; DOI : https://doi.org/10.4000/rao.813 


\title{
Évolution des formes de l'exploitation agricole dans la campagne normande (2500-30 avant J.-C.) L'exemple des fouilles préventives de la périphérie sud de Caen
}

\author{
Evolution of agricultural exploitation types in Norman country (2500-30 BC) \\ The example of preventive excavations in the southern outskirts of Caen
}

\author{
Benjamin Van den Bosche *, Vincent Carpentier ** et Cyril Marcigny ***
}

\begin{abstract}
Résumé : La périphérie de l'actuelle ville de Caen (Calvados) a connu, ces quinze dernières années, un accroissement sans précédent de ses aménagements périurbains (zones industrielles, lotissements, routes, etc.), précédés d'autant d'opérations d'archéologie préventive, diagnostics ou fouilles, parfois sur des surfaces dépassant la dizaine d'hectares. Ce secteur de près de 1400 hectares (dont 500 ha étudiés) apparaît donc comme un terrain d'étude privilégié pour tenter de caractériser les modalités d'occupation de l'espace sur le temps long. L'étude présentée repose sur le développement d'un système d'information géographique (SIG) au sein duquel a été cartographié et renseigné l'ensemble des structures datées du Néolithique final à la fin de l'indépendance gauloise.

Dans le cadre de cette analyse, l'accent a plus particulièrement été mis sur la délimitation chronologique des phases d'expansion et de contraction des domaines ruraux. Les auteurs ont également essayé de quantifier l'intensité de ces phénomènes. La confrontation des données a d'abord permis de mettre en lumière une tendance globale à la densification des occupations. Ce mouvement est toutefois loin d'être linéaire; deux pulsations fondamentales ont été identifiées. La première s'inscrit à la charnière du Bronze moyen et du Bronze final (entre les XV et XII ${ }^{\mathrm{e}}$ siècles avant notre ère). Elle se traduit par la recrudescence d'enceintes domestiques vraisemblablement soumises à des déplacements réguliers au sein de territoires déjà largement ouverts.

Le second épisode marquant débute autour de la deuxième moitié du v siècle avant notre ère pour s'amplifier au III $^{\mathrm{e}}$. Il correspond à une importante réforme des modalités de valorisation du milieu et des moyens de production. L'émergence des " fermes indigènes » s'accompagne d'une géométrisation pérenne des campagnes, d'une différenciation fonctionnelle des sites et de l'affirmation de propriétaires fonciers aisés.

Les acquis portent aussi sur l'organisation spatiale du plateau de Mondeville au i ${ }^{\text {er }}$ siècle avant notre ère. Cet espace rural apparaît alors fortement dynamisé par l'imbrication étroite d'établissements agricoles régulièrement espacés et interdépendants. Les spécificités du système agraire gaulois local, fondé principalement sur l'élevage et les cultures intensives, influent toutefois sur la taille et la structuration des territoires exploités.
\end{abstract}

Abstract: The suburbs of the city of Caen (Calvados, Lower Normandy) have seen an increasing amount of development over the last fifteen years. All this construction work has been preceded by systematic archaeological investigations involving trial-trenching or excavation, the latter often covering several hectares. Out of a total of 1400, 500 hectares have thus been investigated, providing an ideal sample zone for research into the history of settlement patterns. A GIS program, presented in this paper, has been used to record and map all archaeological remains dating from the Late Neolithic to the beginnings of the Roman period.

\footnotetext{
* Doctorant, Université Paris 1 (Panthéon-Sorbonne), UMR 7041-ArScAn - 5 rue Sainte-Anne, 14000 Caen. (benjamin. van-den-bossche@mae. u-paris10.fr)

** Archéologue, INRAP Basse-Normandie, FRE 3119-CRAHAM - 35 Avenue du Président Coty, 14390 Le Hôme-Varaville. (vincent.carpentier@ inrap.fr)

*** Archéologue, INRAP Basse-Normandie, UMR 6566-CReAAH - Le Chaos, 14400 Longues-sur-Mer. (cyril.marcigny@wanadoo.fr)
} 
Our main focus is on the recognition and chronological determination of phases of increase or decrease of farmsteads. The quantification of these phenomena has also been tested. While the combined data for the various periods show an overall trend towards settlement intensification, the progression is not linear. Two striking episodes have been identified. The first occurs at the Middle/Late Bronze Age transition (15 $5^{\text {th }}-12^{\text {th }}$ centuries BC) and corresponds to the emergence of massive domestic enclosures. These settlements, linked to burial sites characterized by major clusters of circular ditched monuments, regularly shifted within a territory that was already cleared of forest.

The second episode probably started during the second half of the $5^{\text {th }}$ century $B C$, and was extended in the $3^{\text {rd }}$ century BC. It coincides with important modifications in exploitation of the environment and in production. Farmstead expansion is associated with geometrical subdivision of space, functional differentiation of settlements and the presence of increasingly prosperous landowners.

Additional information is provided by data on the spatial organisation of the Mondeville plateau during the $1^{\text {st }}$ century BC. This rural area is clearly structured and maintained by a regular network of closely linked and interdependent agricultural settlements. The specificities of the local Gallic agrarian system, mainly based on cattle-breeding and intensive farming, influence the size and layout of the exploited territories.

Mots clés : Analyse spatiale, longue durée, ferme, agriculture, Basse-Normandie.

Key words: Spatial analysis, long term, farmstead, agriculture, Lower Normandy.

Mondeville, Grentheville, Cormelles-le-Royal, Ifs et Fleury-sur-Orne sont des communes périurbaines de l'agglomération caennaise situées sur un bas plateau calcaire qui culmine cependant à près de $70 \mathrm{~m}$ d'altitude. Dominant la rive droite de la vallée de l'Orne, un peu en aval de la confluence avec l'Odon, ces communes s'insèrent parfaitement dans les paysages d'openfield de la Plaine de Caen. Ce vaste corridor de $60 \mathrm{~km}$ de long orienté selon un axe nordsud repose sur des formations calcaires du Secondaire. Il s'apparente à une légère dépression largement ouverte sur le littoral de la Manche et assure la transition entre les ultimes prolongements du Massif armoricain à l'ouest et les assises crayeuses plus récentes qui caractérisent les marges orientales du département du Calvados (fig. 1).

Au sein de cet ensemble, le plateau de Mondeville forme une entité tabulaire assez étendue, délimitée par un large coude de l'Orne. Son inclinaison générale sud-nord est relativement faible. En dépit de cette apparente uniformité, il est recoupé par un certain nombre de talwegs en partie colluvionnés. Le plateau surplombe l'actuelle ville de Caen, installée en contrebas. Ce secteur bas, en position privilégiée de fond d'estuaire et encore fréquemment touché par les crues hivernales, s'inscrit dans une vaste plaine alluviale. Il correspond à une zone d'anciens marais, a priori peu hospitalière mais aujourd'hui totalement urbanisée.

Les sites dont il est ici question se répartissent en cinq zones distinctes correspondant à autant d'opérations d'aménagement à l'intérieur d'un vaste espace industriel et commercial développé en périphérie de ville. Il s'agit, du nord-est au sud-ouest, de la ZAC du MIR (30 ha), de la ZAC de l'Étoile (60 ha), de la ZI de Mondeville Sud (110 ha), de la ZAC Object'Ifs Sud ( 80 ha) et du Parc d'Activité de Fleury-sur-Orne (50 ha). Entre ces zones, de nombreuses opérations de diagnostic et de fouille ont été menées à la faveur d'aménagements plus ou moins ponctuels (routes, lotissements). L'ensemble ainsi exploré couvre une bande de 1400 hectares dont 500 ont été sondés et/ou fouillés depuis les années 1990 (fig. 2; Marcigny et al., 2006).

\section{LES POLITIQUES DE RECHERCHE}

La volonté affirmée par les services de l'État de couvrir, dans ce secteur, l'intégralité des aménagements - quelle que soit leur ampleur - depuis la fin des années 1980, afin d'analyser sur de très grandes surfaces l'ensemble des sites archéologiques a été particulièrement fructueuse au plan scientifique. Cette politique active a créé les conditions d'un renouvellement significatif des connaissances sur l'évolution des sociétés anciennes, depuis l'arrivée des premiers agriculteurs au début du v $v^{\mathrm{e}}$ millénaire avant J.-C. (Chancerel et al., 2006a) jusqu’à la stabilisation des centres paroissiaux médiévaux à compter du viI siècle (Lorren, 1983 et 1989; Taupin, 1996). Elle a ainsi contribué à redessiner les contours d'une histoire locale trop souvent réduite caricaturalement à quelques épisodes glorieux ou douloureux.

Par ailleurs, le fait qu'un même opérateur d'archéologie préventive ait pour l'instant été retenu pour les différentes études d'impact et les fouilles a assuré une plus grande cohérence dans les choix opérationnels et dans la définition de projets de recherche ambitieux en facilitant l'uniformisation des procédures de terrain et d'analyse.

Cependant, les implications administratives des enseignements scientifiques de telles opérations ne semblent pas avoir été complètement assimilées au niveau régional, les arbitrages administratifs les plus récents engendrant un mouvement de 


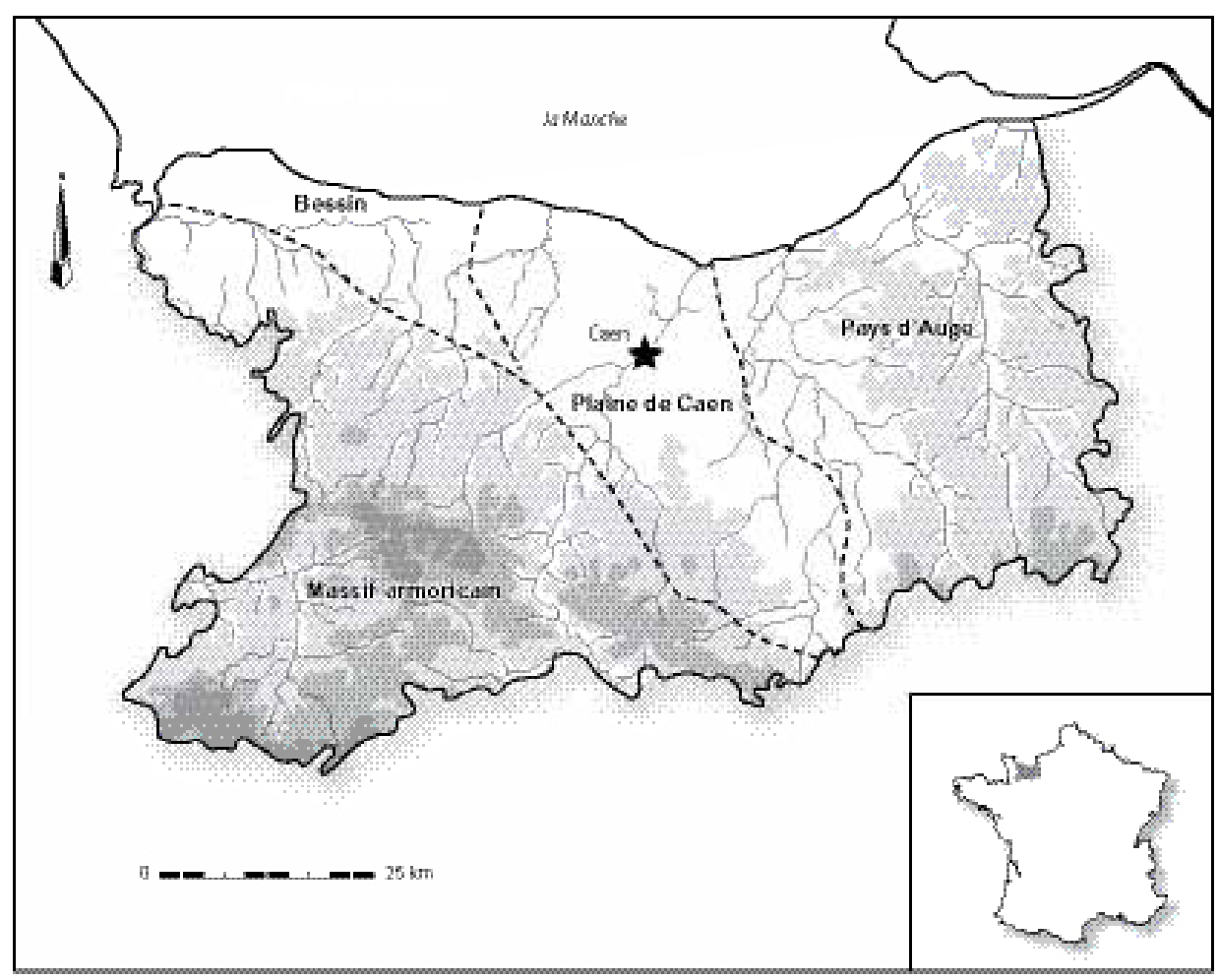

Figure 1 : Topographie et grands ensembles paysagers du département du Calvados.

Figure 1: Topography and main landscape features in the Department of Calvados.

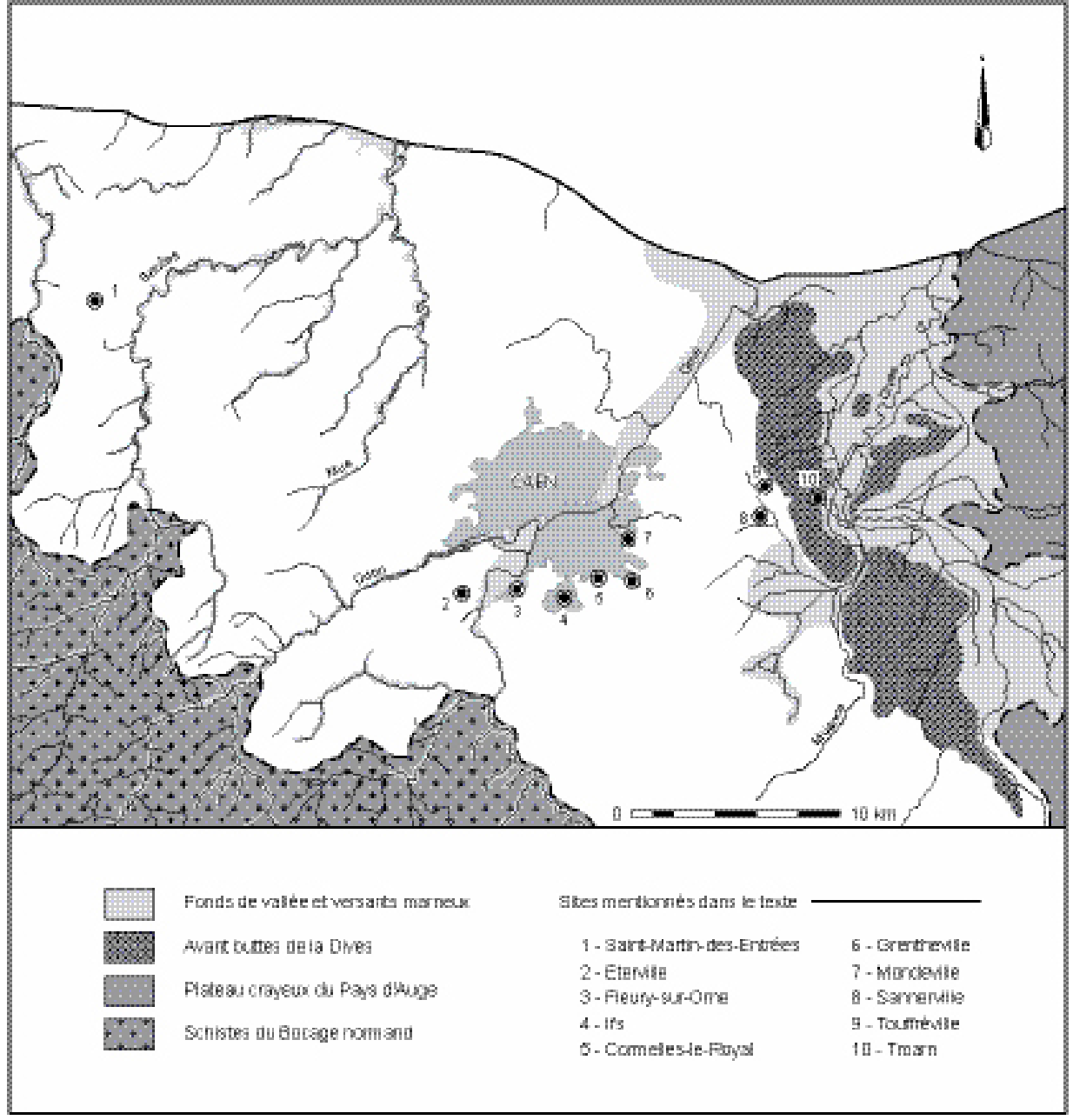




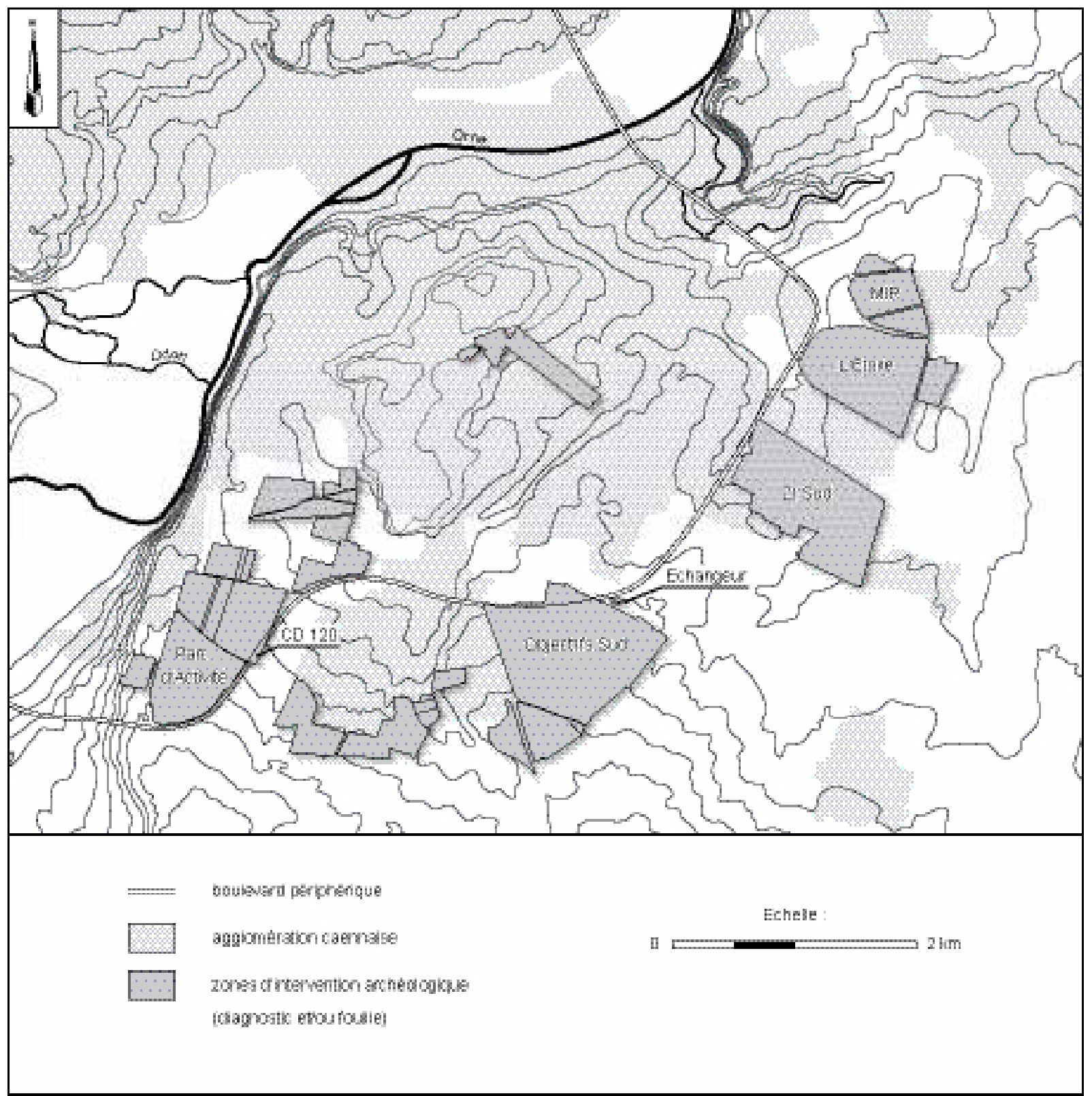

Figure 2 : Localisation des principales opérations d'archéologie préventive récentes en périphérie sud de Caen. Figure 2: Location of the main recent recue archaeology operations undertaken south of Caen.

régression méthodologique regrettable puisqu'ils privilégient les zones les plus riches en vestiges tout en délaissant les espaces interstitiels jugés a priori moins attractifs et peu rentables (Marcigny et al., 2006). Les vertus avérées des décapages exhaustifs n'ont manifestement pas fait fléchir ni réfléchir les décideurs, soumis à des pressions financières croissantes. L'opposition dialectique entre les logiques économiques d'une part et les exigences scientifiques de l'autre ne peut pourtant pas se satisfaire de compromis (Brun et al., 2006). Celui qui consiste à autoriser la réalisation de sondages élargis à l'issue de la phase de diagnostic n'offre par exemple pas toujours les garanties nécessaires à la bonne caractérisation des occupations protohistoriques et antiques. Le risque est donc grand de s'appuyer sur des données fragmentaires, mal maîtrisées, et d'aboutir finalement à une lecture erronée des sites.

L'exemple caennais le montre sans aucune ambiguïté : de tels choix conduisent à la perte irrémédiable d'informations pourtant cruciales dans une perspective d'appréhension globale des sociétés anciennes. L'accumulation des données acquises sur le terrain ces dernières années permet de bénéficier d'une profondeur de champ historique encore inédite 
en Normandie et de disposer d'un degré de résolution chronologique suffisant pour tenter de saisir les rythmes de coévolution des systèmes anthropiques et environnementaux (Burnouf, 2007). Et c'est en privilégiant ce type d'approche diachronique que l'on peut espérer comprendre la dynamique des réseaux de sites sur le temps long et identifier les processus d'interaction entre l'homme et le milieu.

\section{Les rythmes de l'occupation humaine dans la Plaine de Caen (2500-30 av. J.-C.)}

À travers cette étude, qui porte principalement sur l'évolution des modalités d'occupation du sol entre le Néolithique final et la fin du deuxième âge du Fer, il s'agit de restituer la trame des peuplements anciens en associant étroitement les problématiques historique et spatiale. Dans cette optique, nous avons d'abord cherché à mettre l'accent sur la reconnaissance et la délimitation chronologique des phases d'expansion ou de contraction des établissements ruraux.

À l'aide d'un SIG, il a notamment été possible de quantifier l'intensité de ces phénomènes. Naturellement, pour construire la courbe présentée (fig. 3), nous ne nous sommes pas contentés d'effectuer un simple comptage des sites connus par prospection ou fouillés de manière plus ou moins approfondie. Il a aussi fallu tenir compte de la durée intrinsèque de chaque période pour pondérer le nombre des observations et éviter de gommer les disparités les plus significatives. En confrontant les données, étape par étape, on constate une tendance générale à la densification des occupations. Globalement, le nombre moyen de sites sem- ble ainsi augmenter sensiblement au cours des deux derniers millénaires avant notre ère.

Il convient toutefois de rappeler qu'il ne s'agit là que d'une vision schématique d'une réalité certainement plus complexe. Les oscillations repérées sont avant tout des indicateurs de tendance; néanmoins, elles peuvent être considérées comme représentatives des dynamiques anthropiques passées. Deux raisons essentielles peuvent être avancées. D'une part, les opérations d'archéologie préventive s'adaptent aux exigences modernes de l'aménagement du territoire et ne peuvent être soupçonnées d'opérer une sélection arbitraire des sites. D'autre part, il est évident que les processus érosifs actuels ont pu altérer la lisibilité des structures enfouies. Mais leur intensité, relativement limitée dans certains secteurs de la périphérie caennaise (d'après J.-P. Camuzard, in Chancerel et al., 2006a), ne suffit pas à rendre compte de la disparition de sites archéologiques.

Ceci étant établi, l'examen de la courbe révèle deux épisodes marquants. Le premier s'inscrit à la charnière des $\mathrm{Xv}^{\mathrm{e}}$ et $\mathrm{XIV}^{\mathrm{e}}$ siècles avant J.-C. À Mondeville «l'Étoile » et «ZI Sud » apparaissent alors de vastes enceintes fossoyées dont les superficies peuvent atteindre plus de $4000 \mathrm{~m}^{2}$ (fig. 4). Leur rôle exact n'est malheureusement pas connu dans le détail, bien que leur vocation domestique soit pour l'instant privilégiée. Les structures internes, faiblement implantées dans le substrat et sans doute effacées par l'érosion, ne subsistent plus et les quelques reliquats identifiés au cours des fouilles ne fournissent pas de gages suffisants pour leur attribuer une fonction précise. L'analyse de la répartition des mobiliers détritiques retrouvés dans les fossés périphériques confirme
Figure 3 : Évolution de l'occupation du sol dans la périphérie sud de Caen entre le Néolithique final et la fin de la période gauloise.

Figure 3: Evolution of settlement patterns from Late Neolithic to the end of Late Iron Age to the south of Caen.

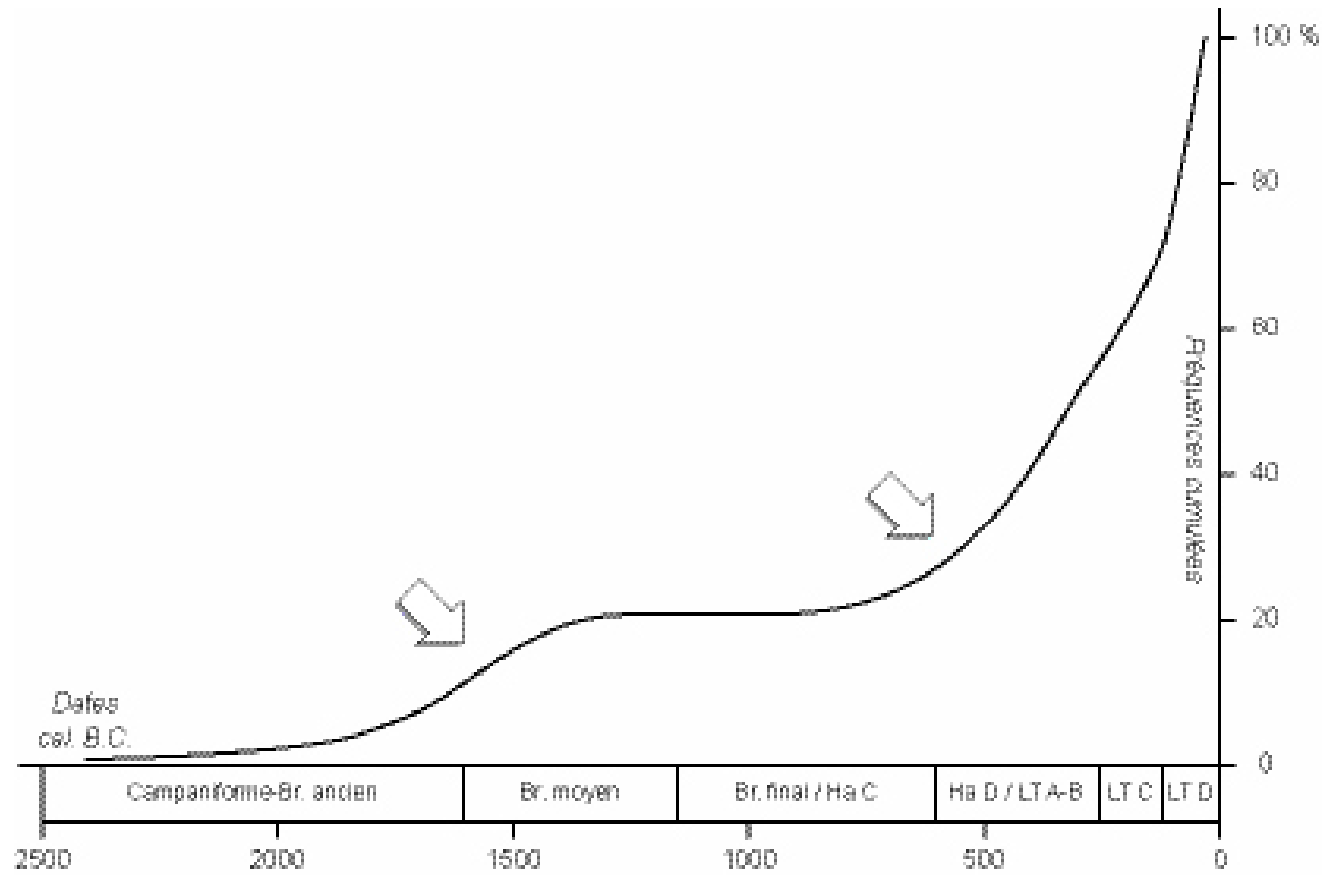

$100 \%$ 


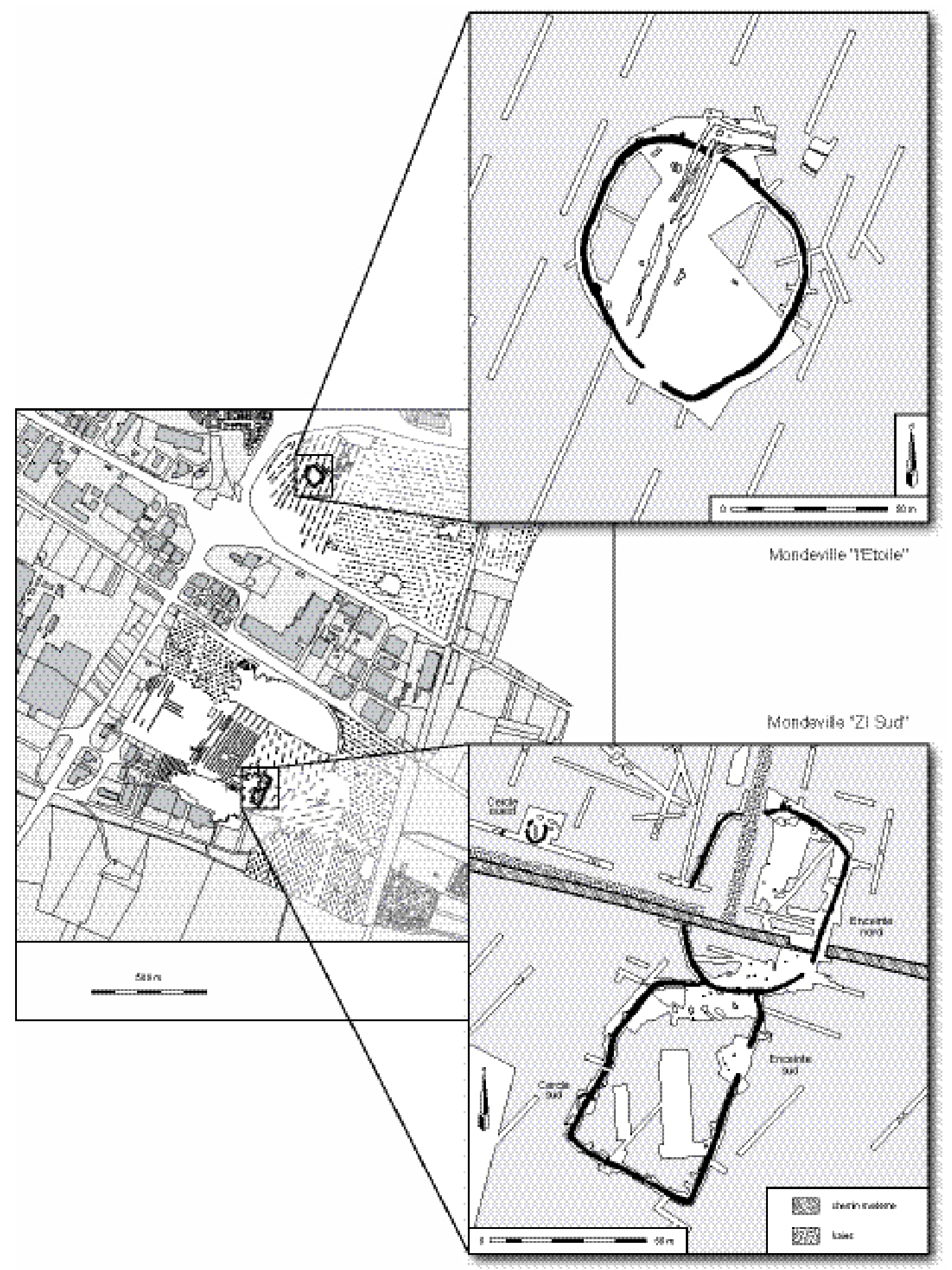

Figure 4 : Localisation et plans des enceintes Deverel-Rimbury de Mondeville « l'Etoile » et " ZI Sud " (d'après BesnardVauterin et al., 2006 et Chancerel et al., 2006b).

Figure 4: Location and plans of the Bronze Age enclosures from Mondeville "l'Etoile" and "ZI Sud". 
toutefois le statut domestique de ces sites : restes osseux, fragments de poteries, meules et pesons témoignent de l'importance des activités de subsistance qui se sont déroulées au sein des aires ainsi encloses (Besnard-Vauterin et al., 2006; Chancerel et al., 2006b).

D'un point de vue chronologique, il semble que ces enceintes n'aient pas toutes strictement fonctionné en même temps; c'est du moins ce que montrent les résultats des campagnes conduites à Mondeville. L'analyse typologique des céramiques exhumées reste l'argument le plus probant en faveur de l'antériorité de l'enceinte de l'Étoile sur celle de la ZI Sud, sans qu'il soit possible d'affiner le phasage étant donné l'imprécision des cadres typochronologiques régionaux (fig. 5; Marcigny et al., 2005). Les datations ${ }^{14} \mathrm{C}$ n'offrent pas d'alternative sérieuse, le pas de temps considéré étant manifestement court.

Les dynamiques de comblement des fossés témoignent en outre de la brièveté de ces occupations. À l'Étoile, la distribution stratigraphique des rejets primaires plaide en faveur d'un abandon rapide du site, alors même que celui-ci était encore potentiellement viable (Besnard-Vauterin et al., 2006). L'absence de traces d'entretien des fossés de l'Étoile comme de la ZI Sud va dans le même sens et semble indiquer que ces sites ont été rapidement abandonnés quel qu’ait pu être le coût de leur réalisation.

À l'échelle du plateau de Mondeville, ces constatations ouvrent toute une série de perspectives en termes d'analyse spatiale. Elles permettent notamment d'envisager l'existence d'occupations successives dans le temps, soumises à des déplacements limités, de l'ordre de $1,5 \mathrm{~km}$. Nous aurions alors affaire à un cas de mobilité relative de l'habitat au sein d'un espace cohérent, modèle déjà mis en évidence en Allemagne ou aux Pays-Bas (Simons, 1989; Fokkens, 1991). Il est effectivement admis que les fermes y opéraient des déplacements cycliques à l'intérieur d'un territoire en suivant l'alternance des parcelles cultivées. Cette pratique conduit à défricher et à valoriser de nouveaux secteurs en fonction d'impératifs encore mal connus. Dans cette optique, l'abandon de l'enceinte de l'Étoile motiverait la fondation d'un autre établissement à peu de distance et selon des modalités équivalentes : superficie de quelques milliers de mètres carrés, entrée unique, éventuels aménagements internes peu ancrés au sol. Ces indices confortent d'ailleurs l'idée d'une proximité chronologique forte entre les deux sites de Mondeville précités et iraient dans le sens d'une continuité de l'occupation assez marquée.

En marge de ces enceintes se développent des ensembles funéraires caractérisés par d'importantes concentrations de monuments circulaires fossoyés, eux aussi très arasés. Ainsi la nécropole de Mondeville "MIR », dont le plan a été perturbé par des installations domestiques postérieures, est-elle constituée de huit enclos circulaires simples autour desquels gravitent une trentaine d'inhumations (Marcigny, 2003). Sur la base de comparaisons régionales et extra-régionales, il semble probable d'attribuer ces structures à la deuxième moitié du II ${ }^{\mathrm{e}}$ millénaire avant notre ère. Cette proposition est corroborée par deux dates ${ }^{14} \mathrm{C}$ qui situent l'utilisation de l'espace funéraire entre le $\mathrm{XVI}^{\mathrm{e}}$ et le $\mathrm{XI}^{\mathrm{e}}$ siècle avant J.-C. (Gif $9194: 3420 \pm 55$ BP soit 1590-1360 BC; Gif $9195: 3080$ $\pm 60 \mathrm{BP}$ soit $1260-1010 \mathrm{BC}$ ).

Ces sites domestiques et funéraires renvoient l'image d'un plateau déjà densément occupé et largement mis en valeur dès le milieu du $\mathrm{II}^{\mathrm{e}}$ millénaire. Cette période d'expansion marque durablement les paysages comme le suggèrent les analyses paléoenvironnementales (Lespez et al., 2004; Marcigny et al., 2007; Carpentier, 2007; Carpentier et al., 2007a). Les défrichements y sont massifs et trahissent l'intensification des activités agropastorales dans la Plaine de
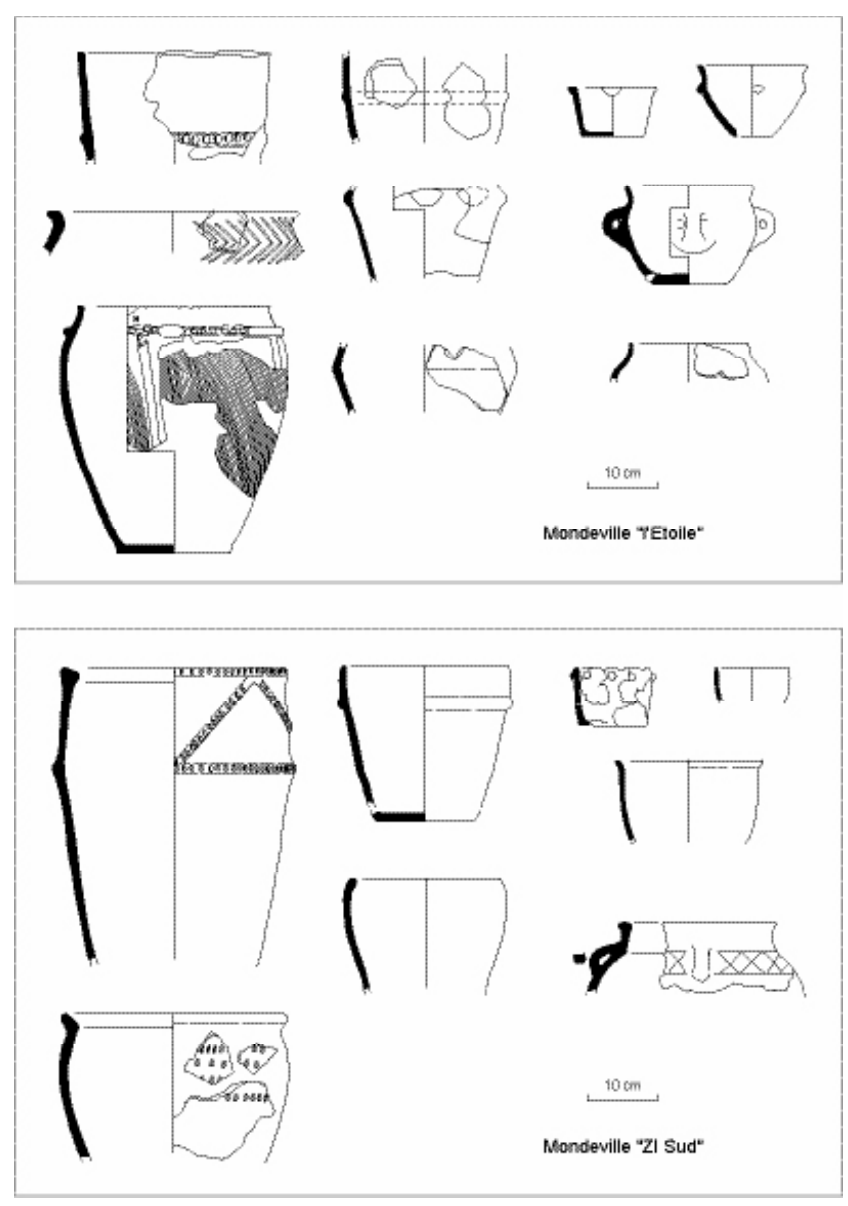

Figure 5: Mobilier céramique de l'âge du Bronze moyen de Mondeville "l'Étoile " et "ZI Sud " (d'après Chancerel et al., 2006a).

Figure 5: Middle Bronze Age ceramics from Mondeville "l'Etoile" and "ZI Sud". 
Caen. Les lacunes documentaires sont toutefois encore trop grandes pour permettre d'affiner l'analyse.

Le second épisode débute vraisemblablement au v $v^{e}$ siècle avant J.-C., c'est-à-dire à la transition entre le Hallstatt D et La Tène A. En effet, la courbe traduit alors une nette accélération du nombre de sites. S'ouvre alors une période de croissance continue très marquée. Cette tendance ne sera d'ailleurs pas contrariée par la suite; elle semble même devoir s'amplifier au cours du III $^{\mathrm{e}}$ siècle avant J.-C.

Ce mouvement correspond à une évolution en profondeur des modalités d'accaparement du milieu et des moyens de production. Il se caractérise par la mise en place d'un paysage agraire structuré, bien ancré dans le sol. Les établissements agricoles datant du deuxième âge du Fer sont tous constitués d'espaces fonctionnellement distincts. Les différents secteurs d'activité y sont matérialisés au sol par des enclos contigus renfermant de nombreuses structures : bâtiments d'habitation, annexes, caves, silos, fours, etc. (fig. 6).

À l'échelle de la zone étudiée, la trame des sites apparaît beaucoup plus dense qu'aux étapes précédentes. Naturellement, tous les établissements n'ont pas fonctionné en même temps, bien que les sites semblent avoir une durée d'occupation plus longue qu'auparavant. Les cartes sont donc trompeuses; elles dénotent cependant une tendance à la fixation progressive des exploitations sur un même terroir. Les enclos subissent certes des réaménagements au cours du temps, mais l'habitat ne se déplace plus au gré de l'ouverture de nouvelles parcelles. Autour de ces enclos s'amorce un véritable découpage géométrique du paysage : les fossés parcellaires appuyés sur le réseau viaire quadrillent littéralement la campagne. Cette organisation marque une nouvelle étape dans l'appropriation du sol et la rationalisation des activités agropastorales. Elle suppose aussi l'adaptation de certaines composantes du système agraire gaulois.

Le semis des sites laténiens observés répond manifestement à des logiques qu'il convient maintenant de préciser. Le nombre des occurrences archéologiques attribuées à la fin du deuxième âge du Fer offre l'opportunité d'engager une étude rigoureuse destinée à tester la validité des différentes variables entrant en jeu dans l'organisation de l'espace sur le plateau de Mondeville.

\section{Le Plateau de MondeVille AUX II $^{\mathrm{E}}$ ET I ${ }^{\mathrm{ER}}$ SIÈCLES AVANT NOTRE ÈRE : ESSAI DE MODÉLISATION}

Avant d'aller plus loin dans cette tentative de modélisation de l'agrosystème gaulois local, il est nécessaire d'introduire un certain nombre d'éléments qui agissent comme autant de paramètres déterminants dans la définition du système cultural des $\mathrm{II}^{\mathrm{e}}$ et $\mathrm{I}^{\mathrm{er}}$ siècles avant notre ère.

\section{Système d'exploitation et système de production}

Le corpus d'outils gaulois découvert en périphérie caennaise demeure relativement maigre. Les problèmes de conservation du mobilier en fer et la récupération du métal sont évidemment responsables de l'indigence de la documentation ett l'inventaire qui a été dressé ne saurait refléter le niveau d'équipement réel des fermes de La Tène finale (d'autant plus que la plupart des sites n'ont été que partiellement étudiés et n'ont pas encore fait l'objet de publication monographique). Néanmoins, les artefacts disponibles constituent probablement une assez bonne illustration des différentes activités qui rythmaient alors le calendrier agricole.

Au total, ce sont un peu plus d'une dizaine d'objets ne posant pas de problème d'identification fonctionnelle qui ont été retenus dans le cadre de cet exercice. Il s'agit le plus souvent d'outils relatifs au travail de la terre d'une part et à la récolte de végétaux de l'autre (fig. 7 et 8 ).

Parmi ces derniers, nous attirons l'attention sur les serpes recueillies à Fleury-sur-Orne "CD 120 " (San Juan et al., 1994); ce sont les seules publiées à ce jour mais des exemplaires similaires existent à Mondeville " Haut-Saint-Martin » et « l'Étoile » où leur présence est loin d'être insignifiante. Ces outils possèdent une fonction bien déterminée, en partie liée à la production animale (Sigaut, 1998). Les exemplaires dont il est question ici témoignent alors d'une probable récolte de feuilles d'arbres comme fourrage d'appoint en prévision de périodes de stabulation plus ou moins longues (Rasmussen, 1990; 1991 et 1993; Malrain et al., 2002). De tels outils renvoient donc l'image d'une pratique de l'élevage bien rodée et laissent envisager l'existence de bâtiments et/ou d'abris spécifiquement dédiés à l'hivernage du bétail ; un fait encore bien difficile à démontrer à la simple lecture des structures archéologiques repérées sur le terrain, mais qu'il conviendrait d'analyser avec le plus grand soin.

Parallèlement, serpes et serpettes participent à la construction des paysages agraires à plus d'un titre. Premièrement, l'émondage suggère l'exploitation partielle d'espaces boisés ou, du moins, d'espaces moins anthropisés, situés à une distance raisonnable de la ferme étant donné l'importance des volumes à transporter. Deuxièmement, ces outils peuvent être impliqués dans l'entretien des haies durant l'hiver (Sigaut, 1998). Ils témoigneraient alors indirectement d'une possible matérialisation verticale des limites parcellaires à l'aide d'espèces arbustives. Celles-ci auraient joué le rôle de clôtures, facilitant par exemple le parcage occasionnel du bétail. Malheureusement, les données paléoenvironnemen- 


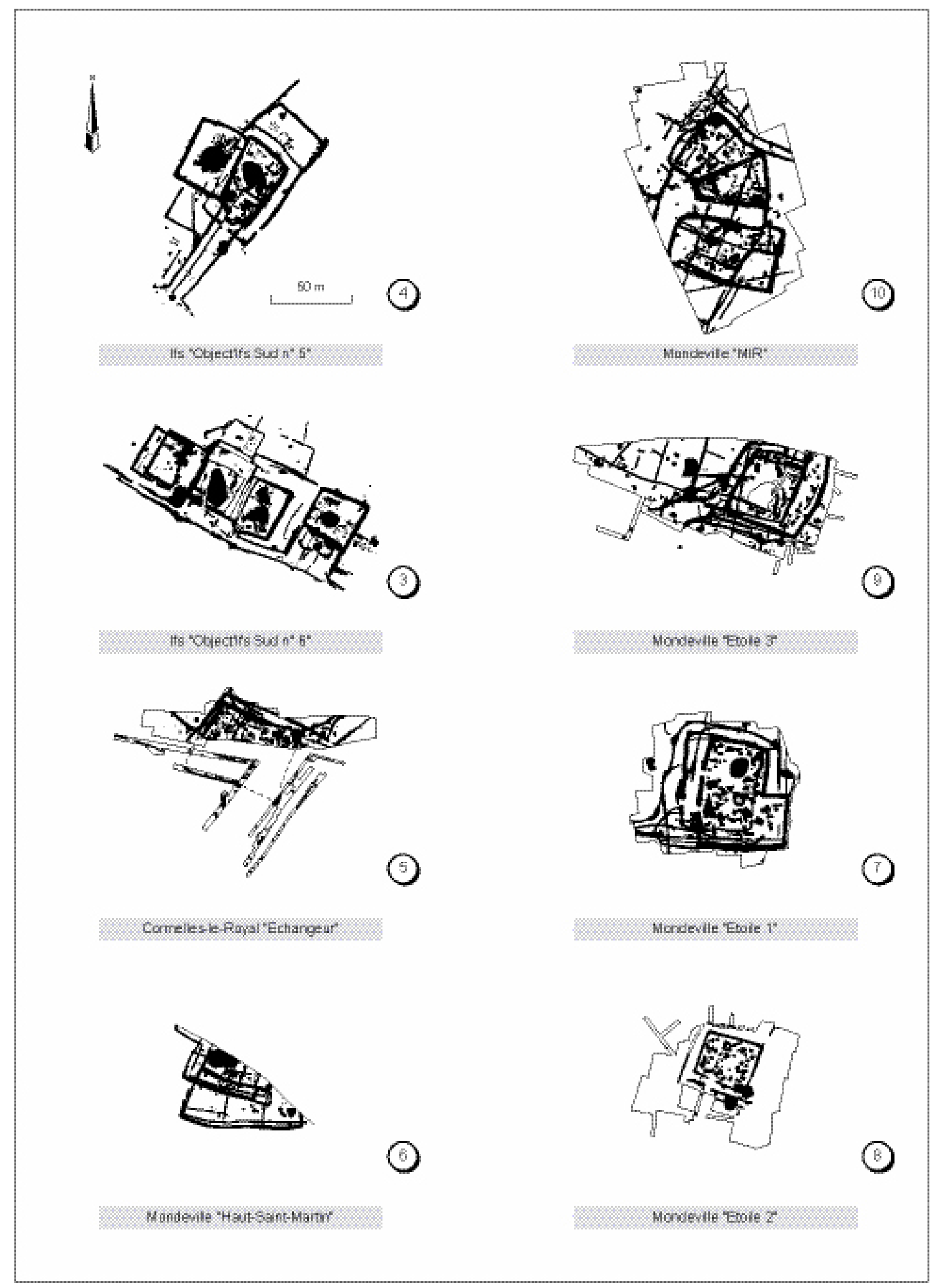

Figure 6 : Plans des établissements agricoles gaulois de la périphérie caennaise (DAO M. Besnard, INRAP; d'après Carpentier et al., 2006, p. 147). Les numéros renvoient à la figure 13.

Figure 6: Comparative plans of the Gallic farmsteads around Caen. Numbers refer to fig. 13. 


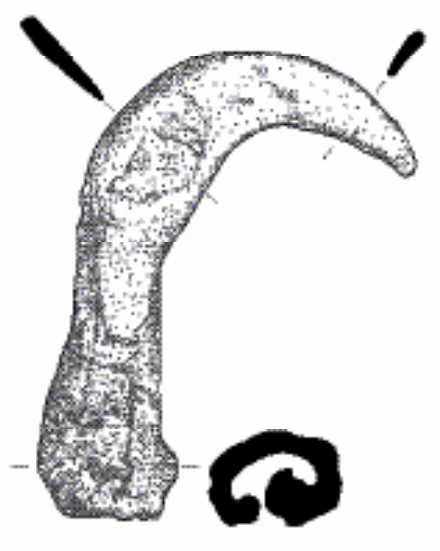

1

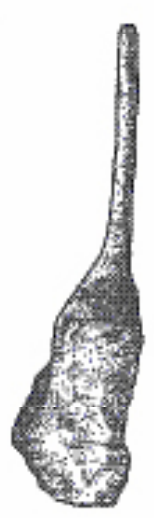

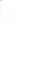

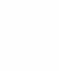
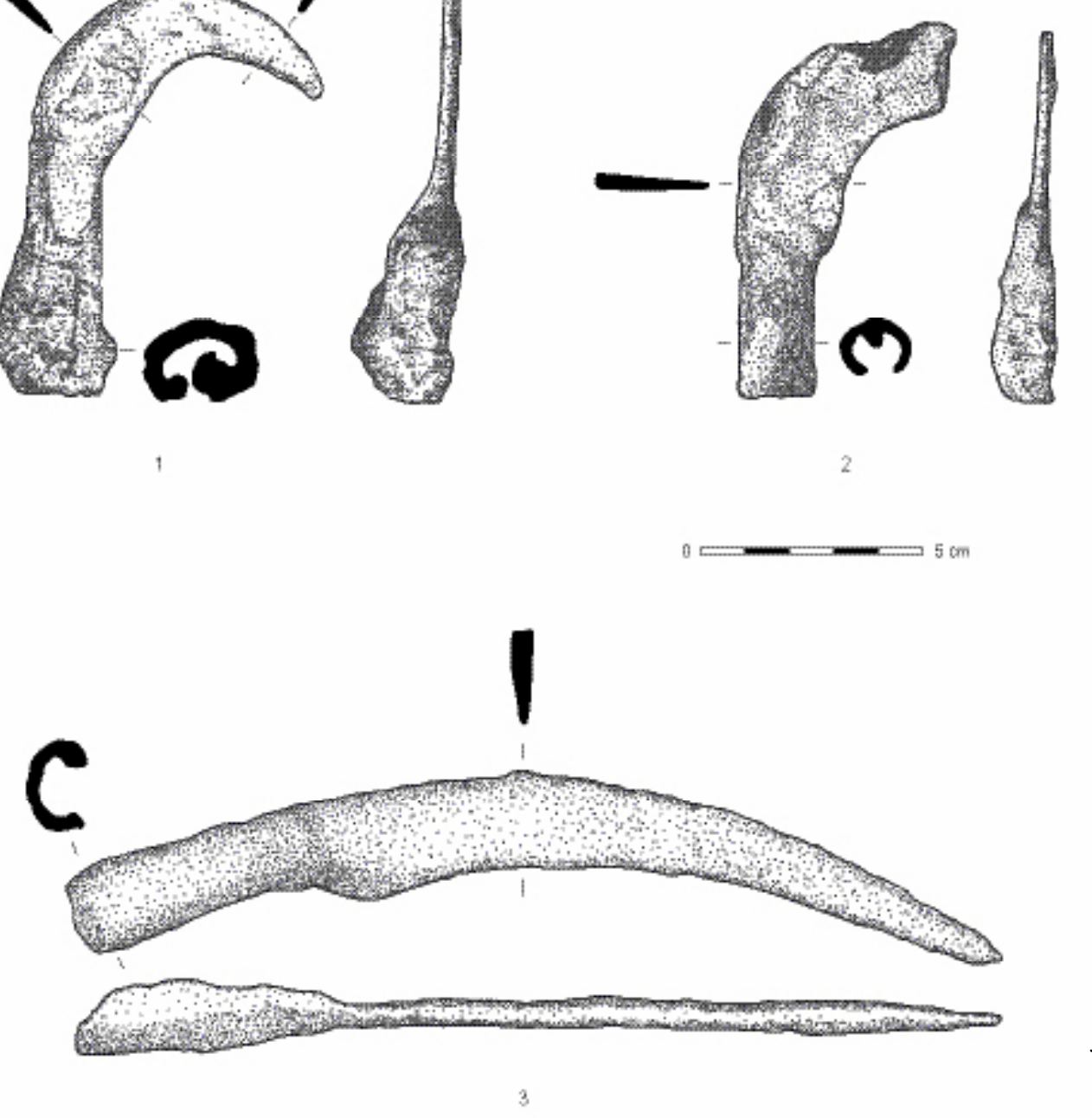

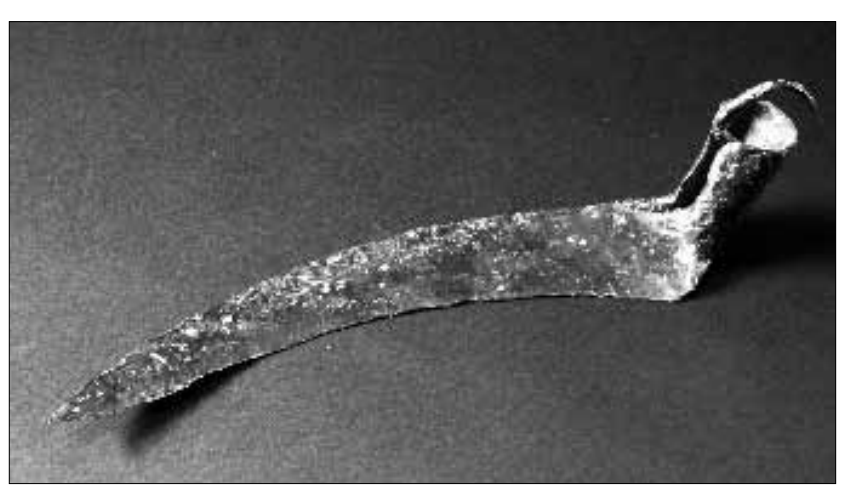

Figure 8 : Lame de faux découverte sur le site d'Ifs « Object'Ifs Sud, ferme $n^{\circ} 5$ " (cliché H. Paitier, INRAP; coll. Musée de Normandie, ville de Caen; d'après Carpentier $e t$ al., 2007b).

Figure 8: Scythe blade recovered from Ifs "Object'Ifs Sud, farm $n^{\circ} 5$. tales sur le secteur sont très limitées et généralement peu significatives (information orale M. Clet-Pellerin, Centre de Géomorphologie de Caen).

Les lames de faux mises au jour à Ifs "Object'Ifs Sud " (Carpentier et al., 2007b) et Fleury-sur-Orne «Parc d'Activité ", (Chanson et al., 2002), de même qu'à Thaon (San Juan et al., 1999) et Caen "ZAC de Beaulieu " (BesnardVauterin et Navarre, 2008), apportent elles aussi de nouveaux éclairages sur le stockage de provisions destinées à compenser le déficit fourrager hivernal et sur l'entretien d'herbages à proximité des établissements gaulois (Ferdière, 1991). Ce dernier point est difficile à démontrer en l'absence de données explicites. Les analyses palynologiques disponibles vont toutes dans le sens de paysages ouverts où se côtoient champs cultivés et prairies (Clet-Pellerin et Verron, 2004; Carpentier, 2007). Les prélèvements ont toutefois rarement concerné les bas plateaux et il serait hasardeux d'en tirer des conclusions définitives. 
Les données accumulées à ce jour dans la basse vallée de la Dives, sur la frange est de la plaine caennaise, revêtent de ce point de vue un caractère exceptionnel. Pour le plateau dominant la rive gauche, dans la région de Touffréville, Troarn et Sannerville, celles-ci confirment l'existence d'un paysage largement ouvert et cultivé, de surcroît de longue date, à la fin de l'âge du Fer, tandis que la transgression marine qui y est ressentie depuis l'âge du Bronze accentue progressivement l'inondation du fond de vallée, avec un maximum atteint entre 400 et 50 avant J.-C. environ (Carpentier, 2007; Carpentier et al., 2007a). Les évolutions observées dans les enregistrements polliniques du fond de vallée révèlent une emprise agraire nettement plus limitée au pied des versants et sur les rives du marais que vers le sommet des plateaux dont l'érosion pédologique gagne en intensité sous la pression agricole, de telle sorte qu'en amont de Troarn, sensiblement à hauteur des sites d'Ifs « Object'Ifs Sud ", la tourbière de Chicheboville-Bellengreville est touchée par un processus d'atterrissement engendré par des apports limoneux à caractère détritique issus de la mise en culture des plateaux voisins. Les marges de la tourbière, aux confins de la Plaine de Caen, sont ainsi touchées par la mise en valeur agricole gauloise attestée par le développement des herbes de prairie, des céréales, rudérales et adventices.

En sus de ces indices palynologiques, la configuration topographique de la zone d'étude nous incite à argumenter en faveur de l'existence de véritables prés de fauche au sommet des plateaux de la plaine caennaise, dans le proche environnement des habitats. Ces prés ont pu se développer dans les vallons aujourd'hui comblés et dans les secteurs légèrement déprimés dont les sols, caractérisés par une hydromorphie marquée, restent sensibles aux fluctuations du niveau de la nappe phréatique, notamment lors d'épisodes climatiques humides. Une telle hypothèse, bien qu'en attente de confirmation, s'accorderait avec le schéma général du développement des espaces prairiaux tel qu'il est aujourd'hui sous-entendu par les archéobotanistes. Ceuxci identifient en effet, dès le cours de l'âge du Bronze en Europe occidentale, l'apparition des flores prairiales anthropozoogènes (cortèges de Molino-Arrhenatheretea - flore de fauche sur sols humides - et de Brometea - flore de pâture sur sols secs), suivie d'un mouvement progressif de diversification de ces mêmes flores entre la fin de l'âge du Fer et l'époque romaine : cortèges de l'Arrhenatheretea et légumineuses fourragères (Ruas, 2008, p. 43).

Dans le cadre de ce questionnement, il serait également intéressant de réévaluer la signification exacte de l'évolution des stratégies d'abattage des bovidés et des caprinés sur le long terme. Les travaux archéozoologiques menés dans ce secteur ont montré une nette régression de l'abattage des animaux juvéniles au cours de la deuxième moitié du second âge du Fer (P. Méniel in San Juan et al., 1994; G. Auxiette in Le Goff et al., 2007). Pour certains chercheurs, les causes de ce recul seraient principalement d'ordre socio-économique : les fermes gauloises seraient victimes d'un déclin relatif au cours du $\mathrm{I}^{\mathrm{er}}$ siècle avant notre ère; leurs occupants ne pourraient alors plus se payer le luxe de consommer de la viande de qualité. Ce type d'interprétation s'accorde pourtant difficilement avec les autres données matérielles qui trahissent plutôt l'aisance des propriétaires des lieux. C'est du moins ce que suggère le dépôt monétaire mis au jour dans le comblement de l'un des fossés d'enclos de la ferme $\mathrm{n}^{\circ} 5$ d'Ifs « Object'Ifs Sud ». Daté du milieu du i ${ }^{\text {er }}$ siècle avant notre ère, cet ensemble composé de 33 monnaies d'or et d'argent ainsi que de 34 potins indique la relative prospérité des occupants (Le Goff et al., 2007).

À la lumière de ces éléments, peut-être faudrait-il plus simplement renverser la proposition et comprendre cette tendance évolutive comme le reflet de nouvelles pratiques rendues possibles par le développement de vastes superficies dédiées à la récolte des foins. Dans un système où les disponibilités fourragères sont faibles, les agriculteurs sont effectivement obligés d'abattre, à l'approche de la mauvaise saison, une partie des animaux nés au printemps afin de garantir la survie du reste du troupeau (Mazoyer et Roudart, 1997). La forte représentation des très jeunes individus dans les différents profils d'abattage ne serait donc pas uniquement liée à des considérations gustatives et hiérarchiques; elle serait aussi en partie dépendante de la fragilité des agrosystèmes archä̈ques, incapables de répondre aux besoins alimentaires d'un troupeau quelque peu important durant l'hiver.

Avec la multiplication des prés de fauche, cette contrainte devient moins prégnante, voire obsolète. Les risques de pénurie en fourrages se trouvent minimisés et la taille du cheptel potentiellement accrue. L'examen des courbes d'abattage montre une réorientation sensible des modes de gestion du bétail au cours de La Tène finale : dans leur grande majorité, les bœufs et les moutons font l'objet de soins particuliers; ils sont maintenus en vie jusqu'à des âges assez avancés sans toutefois dépasser le seuil de rentabilité bouchère. L'exploitation de la viande n'est donc plus l'unique objectif des paysans. Ceux-ci sont peut-être également préoccupés par la constitution d'abondants stocks de fumier sans lesquels l'augmentation de la production agricole n'est pas possible. Dans ce contexte, il devient en effet nécessaire de mettre en place des mécanismes facilitant la régénération des sols cultivés. 


\section{Réflexions sur le renouvellement de la fertilité des terres à l'époque gauloise}

Faute de preuves convaincantes et de données archéologiques explicites, ce sujet reste encore débattu, bien que quelques réticences aient été levées pour des périodes plus anciennes ou pour des territoires bien différents (Troels Smith, 1984; Bakels, 1997; Nuninger, 2002, p. 161-163). Il ne fait pourtant aucun doute que le recours à des techniques permettant le renouvellement de la fertilité des sols ait constitué une caractéristique majeure du système agraire gaulois; du moins peut-on l'envisager à la lumière de plusieurs indices.

Parmi ceux-ci, la présence récurrente de vastes structures faiblement excavées et localisées au centre des enclos domestiques a fait couler beaucoup d'encre (fig. 9). Dans certains cas bien documentés, il s'agit de carrières utilisées pour l'extraction de limons. L'examen des profils de ces

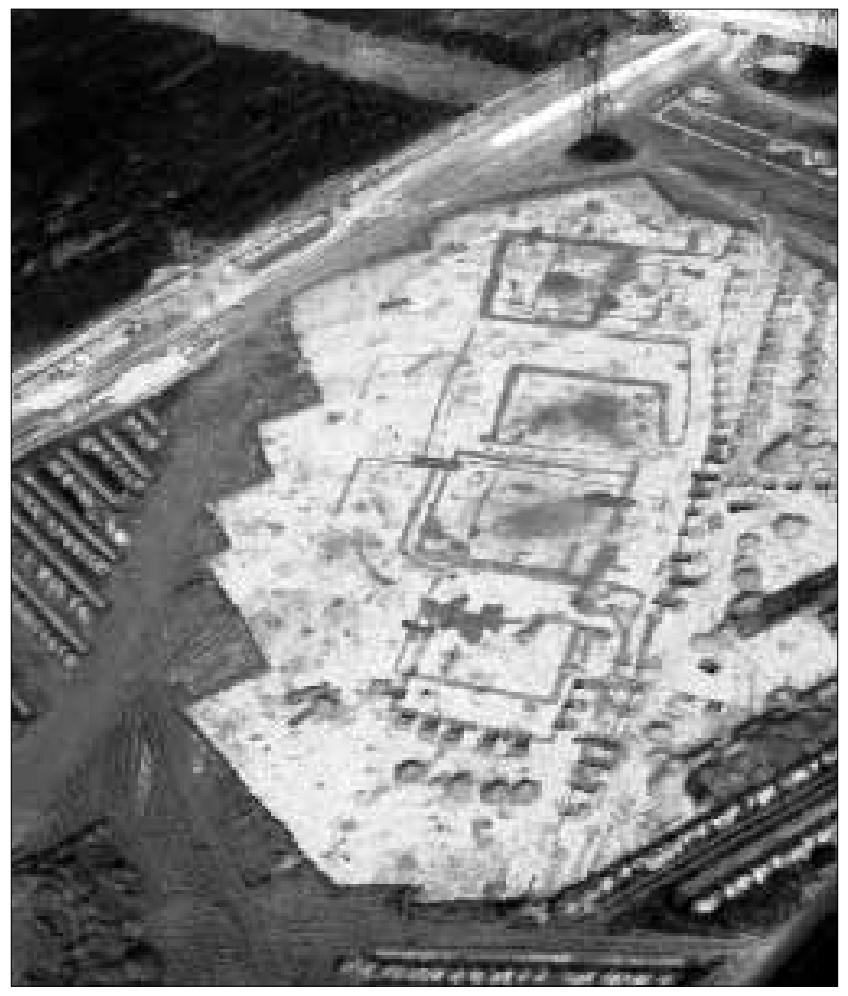

Figure 9 : Vue aérienne de la ferme n 6 d'Ifs « Object'Ifs Sud » en cours de fouille. Trois des enclos domestiques présentent en leur centre des anomalies correspondant à de larges creusements dont la fonction initiale est mal connue; il peut s'agir de fosses d'extraction, de mares ou de fosses à fumier (cliché J. Desloges, DRAC/ SRA Basse-Normandie; d'après Carpentier et al., 2007b).

Figure 9: Ifs "Object'ifs Sud": Air photography of farm $n^{\circ} 6$ during the excavations. Three domestic enclosures are characterized by wide excavated anomalies in the centre, the function of which remains uncertain (extraction pits, ponds or manure pits?). dépressions (moins de 0,50 $\mathrm{m}$ de profondeur en général) a en effet révélé l'existence de plusieurs creusements successifs, entrepris au fur et à mesure des besoins. Dans d'autres cas avérés, il s'agit de mares, comme l'attestent notamment les niveaux hydromorphes visibles en stratigraphie sur la structure de Mondeville "Haut-Saint-Martin " (Marcigny, 1995). Quelques structures demeurent quant à elles davantage problématiques. Suivant en cela divers témoignages à valeur ethnographique, nous serions tentés de les interpréter comme des fosses à fumier, recueillant les déjections animales et les détritus générés par les activités humaines. À titre d'exemple, les descriptions fournies par E. Zola (1889) sur la base des enquêtes qu'il a réalisées dans la campagne beauceronne à la fin du XIx ${ }^{e}$ siècle, constituent une source facilement exploitable pour étayer cette hypothèse. Naturellement, elles ne sont pas exhaustives, mais semblent pertinentes pour deux raisons essentielles : d'une part, elles ne contredisent pas les observations des géographes et semblent même pouvoir être généralisées à l'ensemble des grandes plaines qui composent le bassin Parisien (Hitier, 1903; Servais, 1925); d'autre part, elles mettent en avant l'étendue de ces dispositifs et leur position centrale à l'intérieur des fermes : « [La] fosse à fumier tenait un tiers de la cour. [Françoise] regardait sans pensée une bande de poules, piquant du bec et se chauffant les pattes sur cette large couche basse." (Zola, 1889, p. 8). La superficie occupée par la fosse à fumier mérite d'autant plus notre attention que la Beauce, pays de grandes cultures, reste faiblement concernée par l'élevage (Heuzé, 1875; Poitou, 2007). Il y a donc fort à parier que le système gaulois bas-normand, tourné vers l'exploitation d'un cheptel relativement important, ait conduit à l'aménagement d'installations au moins similaires.

D'emblée, cette hypothèse entrerait effectivement en concordance avec l'absence constatée de toute installation de type étable dans la région normande, en raison de conditions climatiques suffisamment clémentes pour permettre la croissance de l'herbe tout au long de l'année et donc l'hivernage extérieur du bétail (Grundy, 1970; Zimmermann, 1999a, p. 138 et fig. 3). Or ce type de situation induit certaines implications zootechniques non négligeables dans le système agraire, notamment sous l'angle du rapport entre biomasse fourragère et production d'engrais animaux (Barker, 1999). Dans les régions d'Europe occidentale situées au sud de la Mer du Nord, l'une des alternatives les plus répandues à la maison-étable propre aux pays septentrionaux dotés d'un climat rigoureux est "l'enclos à fumier " (dung-yard) auquel W. H. Zimmermann a consacré plusieurs études minutieuses (Zimmermann, 1999a, p. 139; 1999b, p. 298). Les exemples archéologiques et ethnographiques réunis par ce chercheur montrent en effet que ce type de parc à bestiaux, destiné à réunir le bétail et en particulier le cheptel bovin en 
période hivernale, de manière à lui fournir plus facilement, après les opérations de sélection et d'abattage, les compléments fourragers nécessaires à son alimentation, permettait également de collecter à peu de distance de l'habitation de précieux engrais indispensables à la fumure des champs cultivés. En outre, la conduite des troupeaux sur les emblavures après récolte, pratique encore largement attestée jusqu'à une époque récente en Europe occidentale, permettait d'engraisser les champs tout en pourvoyant à la nourriture des animaux grâce aux regains qui croissent au milieu des chaumes (Bloch, 1968, p. 24).

Depuis la Protohistoire jusqu'à l'époque moderne, la production bovine régionale se déploie en effet dans le cadre d'un élevage mixte, conduit en semi-liberté selon un rythme saisonnier aux dépens de la végétation disponible au sein et à la périphérie des terroirs de plateaux. Les données palynologiques réunies dans la basse vallée de la Dives (Carpentier, 2007), une aire fortement spécialisée dans l'élevage bovin à partir du XvII ${ }^{\mathrm{e}}$ siècle, suggèrent que le grand bétail fréquentait déjà les versants de la basse Dives au Bronze moyen, contribuant à l'ouverture des massifs forestiers et surtout des aulnaies attestées sur les rives du marais. Aux $\mathrm{I}^{\mathrm{er}}$-III ${ }^{\mathrm{e}}$ siècles de notre ère, les signaux environnementaux enregistrés au niveau de la villa de Touffréville (Ponel et al., 2000), implantée en rebord du plateau sur la lisière orientale de la Plaine de Caen, confirment bien une importante dépaissance à proximité du site, vraisemblablement tournée vers le pied du versant en période estivale et vers la forêt adjacente en période de soudure, voire durant l'hiver. On ignore en effet totalement dans la région l'existence de grands édifices de stabulation comparables à ceux de l'Europe septentrionale à partir de l'âge du Fer et il convient donc d'envisager la conduite de l'élevage en aire libre, conduite marquée à l'entrée et au sortir de l'hiver par des regroupements et parcages qui étaient vraisemblablement effectués à proximité voire au sein même des habitats (Barker, 1999). Constatons par ailleurs à quel point les établissements enclos qui se répandent dans la région à partir de l'âge du Bronze se prêtent à de telles pratiques. En ce qui concerne les "fermes indigènes " de l'âge du Fer, il semblerait que la distribution périphérique des constructions et aménagements divers (greniers, fosses, fours, souterrains, etc.) à l'intérieur de l'aire enclose puisse découler de cette nécessité de disposer d'un parc à bestiaux utilisable lors des saisons d'abattage et de tri des animaux. Il est probable par ailleurs que ce parcage permettait de produire et recueillir des engrais naturels destinés à l'amendement des cultures de l'infield - une préoccupation de premier plan que l'on retrouve systématiquement évoquée dans les documents écrits concernant la vallée de la Dives, et jusqu’à une époque récente (Carpentier, 2007, en particulier t. IIIB, $\left.\mathrm{n}^{\circ} 83\right)$. Le plan des établissements gallo- romains dotés d'une ou plusieurs cours fermées, à l'instar de la villa de Touffréville, témoignerait peut-être de la pérennité de telles pratiques jusqu’à la fin de l'Antiquité et bien au-delà, pratiques dont les origines paraissent remonter au moins à la période gauloise.

Ce type de gestion consistant à regrouper les animaux au centre ou à proximité de l'habitat dans les limites de parcs spécifiques a pu être mis en évidence par l'étude palynologique de quelques sites du nord-ouest de l'Europe bénéficiant de conditions de conservation peu communes, à l'instar de l'habitat de Flögeln, en basse Saxe (Zimmermann, 1977; Behre et Kucan, 1994, p. 147-152). D'autres identifications ont été proposées dans le secteur des Terpen (PaysBas) ou Wurten (Allemagne), tertres insulaires bordant les rivages de la Mer du Nord, à l'instar de l'habitat de Elisenhof (Schleswig-Hosltein) qui, au début du Moyen Âge, était flanqué d'une vaste terrasse émergée, comprenant une mare abreuvoir et cernée de fossés doublés par des talus renforcés de haies d'épines, dressés de main d'homme (Bantelmann, 1975, p. 55-56; Lebecq, 1980). Ce portrait coïncide en effet avec les illustrations de "dung-yards" recensées par W. H. Zimmermann dans divers ouvrages du XIX ${ }^{e}$ siècle et jusqu'aux années 1950 (Zimmermamm, 1999a, p. 139 et fig. 1). Au I ir siècle avant J.-C., Columelle décrit en outre de tels enclos à bestiaux en plein air, coexistant avec les grandes étables des villae (De Agricultura, I, 6 et IV, 6). Virgile et plus tard Bède le Vénérable y font également allusion (Zimmermann, 1999b, p. 300-301). En Europe du nord (Pologne), l'hivernage extérieur des bovins fut une pratique courante jusqu'au Moyen Âge (Zimmermann, 1999b, p. 304-305). Il semble que l'on ait parfois eu recours à des abris rudimentaires pour protéger les animaux; c'est en tout cas ce que décrit Palladius au $v^{\mathrm{e}}$ siècle de notre ère, en évoquant de tels dispositifs contre les intempéries (Opus Agriculturae, I, 22). Suivant W. H. Zimmermann, le mode de fonctionnement de ces "dung-yards" peut être résumé ainsi : l'aire de piétinement des bestiaux, où s'accumulent leurs déjections, est régulièrement recouverte de lits de foin. Tous les ans, au sortir de la période de parcage, le fumier est retiré et le sol creusé, parfois jusqu'à $1 \mathrm{~m}$ de profondeur, afin de collecter le sédiment riche en nutriments. De la terre est ensuite rapportée afin de combler la fosse (Zimmermann, 1999a, p. 140). Force est de constater combien ce schéma permettrait d'expliquer la stratigraphie complexe des « cuvettes » formées au centre des enclos gaulois de la Plaine de Caen et comblées par une couche de limon vierge, le plus souvent peu ou non hydromorphe, rapportée dans la dépression a posteriori semble-t-il de son utilisation initiale, ce dont témoigne la présence récurrente de quelques fours aménagés aux dépens des parois, en deçà du remplissage limoneux. 
Évidemment, il ne s'agit là que d'une hypothèse reposant sur de faibles indications puisées dans les référentiels ethnographiques et historiques. L'importance des niveaux organiques riches en rejets céramiques et fauniques observés en coupe ne constitue pas en soi une preuve suffisante et pourrait même alimenter d'autres explications. Faute d'analyses chimiques (phosphates), phytologiques et parasitologiques, il n'est pour le moment pas possible d'aller plus loin. Néanmoins, cette hypothèse est à garder en mémoire, d'autant que les conséquences d'une telle proposition sont loin d'être négligeables. Ce type de dispositif suppose en effet des transferts de matière organique depuis la ferme jusqu'aux parcelles cultivées, c'est-à-dire une dépense d'énergie supplémentaire susceptible de modifier sensiblement la configuration spatiale de l'ager. Il implique également l'abandon partiel du transport sur bât, globalement peu rentable, au profit de moyens de transport plus sophistiqués dont l'existence semble confirmée par la densité du réseau viaire reconnu en marge des enclos domestiques.

Le poids de l'élevage au sein des établissements de la périphérie caennaise n'est plus à démontrer. Si les animaux sont avant tout mobilisés pour la production de viande (San Juan et al., 1994; Le Goff et al., 2007), ils représentent également une source potentielle de fumier non négligeable, surtout si l'on admet une stabulation du bétail durant les quelques mois d'hiver. L'état de la documentation ne permet toutefois pas de confirmer ce qui n'est, pour le moment, qu'une supposition. Comme on l'a souligné plus haut, l'exploitation du foin n'est pas forcément corrélée à la présence d'étables : les fourrages peuvent être stockés et consommés en plein air (Zimmermann, 1999; Malrain et al., 2002).

Par ailleurs, aucun des bâtiments fouillés ne présente de spécificité architecturale susceptible de fournir des renseignements sur sa fonction passée. Testées sur l'ensemble des édifices reconnus dans le secteur, les approches typologique et dimensionnelle ne sont pas suffisamment discriminantes. Elles autorisent seulement la partition de l'échantillon en deux sous-ensembles numériquement déséquilibrés. D’un côté, les constructions les plus vastes, systématiquement localisées à l'intérieur des enclos, sont interprétées comme des maisons (Jahier et al., 2000). De l'autre, se trouve un groupe de bâtiments plus nombreux au sein duquel il est bien difficile d'opérer des distinctions fines. Il s'agit dans la plupart des cas d'annexes agricoles, mais en l'absence de sols archéologiques conservés, nous ne sommes pas en mesure de différencier greniers, fenils, granges, étables ou bergeries. Il est toutefois évident que certains établissements ruraux étaient spécialisés dans l'élevage comme en témoignerait le site de Mondeville « l'Étoile I, site 1» (phase 2) où l'enclos principal est précédé, d'une part, d'un dispositif fossoyé potentiellement destiné à canaliser les troupeaux et à faciliter leur gestion au quotidien, et d'autre part, d'un petit enclos annexe éventuellement utilisé pour parquer le bétail (fig. 10) (Lepaumier et al., 2006 d'après les fouilles de C. C. Besnard-Vauterin).

Enfin, la pratique de la pâture en plein champ est attestée par de récentes études portant sur la culture de l'avoine (Avena sativa) en France septentrionale et en contexte laténien. Les analyses phytosociologiques réalisées par V. Matterne sur les corpus bas-normands ont montré le caractère quasi exclusif de la coupe haute (Matterne in Marcigny et al., 2004); autrement dit, les tiges étaient laissées sur place à l'issue de la moisson. L'enfouissement du chaume ayant des résultats discutables en terme de fertilisation des sols (Ferdière et al., 2006), les parcelles devaient être directement pâturées par le bétail, celui-ci pouvant être maintenu sur place à l'aide de dispositifs légers comme des claies mobiles. Ce système permettait non seulement de nourrir les bêtes à moindre coût, mais aussi d'assurer la fumure du lopin de terre concerné. Décrivant le fonctionnement des domaines ruraux du $\mathrm{I}^{\text {er }}$ siècle avant notre ère en Italie, Varron (Res Rusticae, II, 2, 12) indique d'ailleurs que " dans un champ récemment moissonné, la présence d'un troupeau est doublement avantageuse. Il s'engraisse des épis tombés; et, par le fumier qu'il y dépose, mêlé à la paille

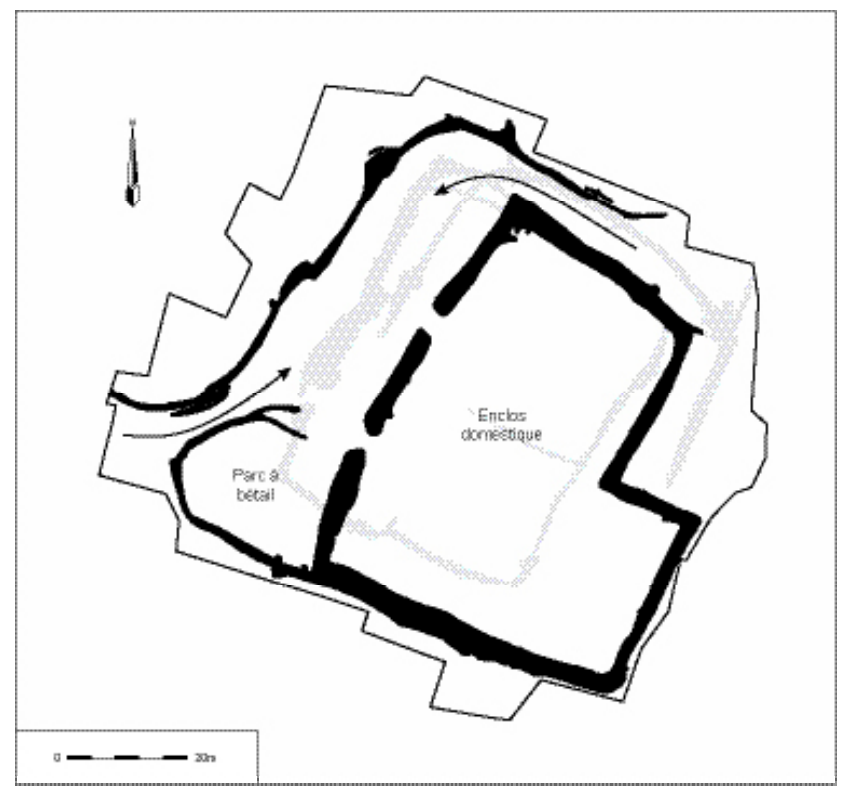

Figure 10 : Organisation interne du site gaulois de Mondeville "l'Étoile 1 "; les vestiges à vocation pastorale et attribués à la deuxième phase d'occupation figurent en noir (d'après Lepaumier et al., 2006, p. 60).

Figure 10: Mondeville "l'Etoile 1": spatial organisation of the site and functional interpretation of the main areas involved in cattle breeding in the second occupation phase. 
broyée sous ses pieds, la terre se trouve amendée pour une récolte à venir ». Il semble que cette pratique, prônée par les agronomes et jugée efficace par les cultivateurs, ait perduré jusqu'à des périodes récentes (Zimmermann, 1999; Zola, 1889, p. 247 ; cf. également divers exemples sur la longue durée réunis dans Brumont, 2008).

Les données recueillies soulignent de manière évidente la place essentielle qu'occupait l'élevage dans l'agrosystème gaulois bas-normand. Plus exactement, la fin du $\mathrm{II}^{\mathrm{e}}$ siècle avant J.-C. semble coïncider avec un renforcement sans précédent des interactions entre céréaliculture et élevage; il ne s'agit plus de deux sous-systèmes parvenus chacun aux limites de leurs capacités et plus ou moins bien articulés entre eux. Un seuil qualitatif est manifestement franchi au cours de La Tène finale; nous aurions alors affaire à deux facettes d'un même système dont la réorganisation en vue d'une meilleure intégration provoque une amélioration des niveaux de production. Naturellement, ces mutations affectent aussi l'organisation spatiale du plateau de Mondeville, qu'il s'agisse des structures foncières ou des modalités d'utilisation du sol.

\section{La construction du modèle}

Le protocole permettant l'élaboration d'un modèle d'utilisation du sol combine des informations environnementales et archéologiques; il peut se décliner en plusieurs étapes.

Dans un premier temps, il convient d'acquérir et d'intégrer des données relatives à l'environnement local actuel en croisant des documents cartographiques avec des informations plus ponctuelles, recueillies sur les chantiers archéologiques eux-mêmes ou issues d'autres ressources (Diomard et al., 2006). Très rapidement, il est apparu que le relief, particulièrement peu accentué au sud de Caen, n'avait pas freiné la mobilité ni l'activité économique des populations installées entre Mondeville et Fleury-sur-Orne. Aussi la définition d'entités physiographiques s'est-elle essentiellement appuyée sur la nature du substrat telle qu'elle est reportée sur les cartes géologiques à $1 / 50000$ et sur la présence de cours d'eau incisant le plateau. Partant de ce postulat, et en mettant l'accent sur les principales propriétés discriminantes des sols rencontrés dans la zone d'étude (texture, structure, hydromorphie, pierrosité), il a été possible de créer trois classes de terroirs.

- Terrains calcaires : il s'agit de sols filtrants à texture argileuse. Peu sensibles à l'hydromorphie, ils sont caractérisés par une proportion de cailloux très variable et conservent un très bon potentiel agronomique (Amiet et al., 1996).

- Colluvions : il s'agit de dépôts de versant comblant d'anciens vallons, aujourd'hui inactifs. Leur texture est le plus souvent limoneuse à limono-argileuse (Lautridou et al.,
2000). L'excès d'eau reste la principale contrainte de ces sols; ils sont par conséquent difficiles à travailler et peu adaptés à la céréaliculture. En revanche, leurs propriétés en font d'excellents terrains pour le développement d'aires de pacage.

- Terrains loessiques : ces terrains, repris par l'érosion antéholocène, présentent une texture limoneuse caractéristique. Les placages de loess carbonatés, plus ou moins épais en fonction de la topographie sous-jacente, possèdent d'excellents potentiels agronomiques. Ils restent néanmoins sensibles à la battance (Amiet et al., 1996).

Cette classification, même si elle reste schématique et discutable, constitue néanmoins un préalable nécessaire à toute analyse. Sur la base de données actuelles considérées comme stables, elle rend compte d'un environnement globalement peu contraignant au sein duquel les problèmes hydrogéologiques demeurent primordiaux. C'est dans ce cadre particulier que s'insèrent des activités agricoles, en partie déterminées par les caractéristiques intrinsèques du sous-sol.

Il faut logiquement tenter d'évaluer cet environnement en fonction de critères adaptés aux activités agricoles. À ce stade, il s'agit d'attribuer à chaque type de sol une valeur de rendement en confrontant les exigences des différentes activités agricoles aux aptitudes des entités définies précédemment et en tenant compte des moyens de production de l'époque (Coste et al., 1988; Thouvenot et al., 2000 ; Vannière, 2001). Les sols lourds, marqués par l'hydromorphie, ont été considérés comme peu adaptés au travail de la terre étant donné le caractère rudimentaire et la sensibilité à l'usure des instruments aratoires gaulois (Sigaut, 1998; Malrain et al., 2002; Serneels, 2007). À l'inverse, ces mêmes sols, sans doute temporairement inondés lors d'épisodes humides saisonniers (automne et fin de l'hiver) semblent parfaitement appropriés pour l'élevage et le développement de prairies (Malrain et al., 2002). Des terrains plus éloignés, placés au contact du plateau et de la vallée de l'Orne, auraient également pu être valorisés dans le cadre de parcours journaliers impliquant les caprinés, ou saisonniers pour les bovins, à l'instar de ce que l'on relève pour l'Antiquité dans de nombreuses régions limitrophes de zones humides et/ou littorales en Europe du Nord-Ouest (Rippon, 2000a et b; Meddens et Beasley, 2001). Ces pratiques supposées ont suscité un important argumentaire dans le cadre de la basse vallée de la Dives, qui vaut aussi pour d'autres secteurs régionaux dont la basse vallée de l'Orne et l'ensemble des zones littorales du Cotentin, de la baie du Mont Saint-Michel à la baie des Veys (Carpentier, 2007). Les autres formations superficielles présentes en Plaine de Caen se prêtent à tous les modes de faire-valoir agricole, à l'exception peut-être des sols loessiques dont la structure a tendance à se dégrader rapidement en cas de piétinement excessif par les animaux (Amiet $e t$ al., 1996). 
L'examen des cartes établies à partir du recensement exhaustif des structures archéologiques met en évidence la densité exceptionnelle des établissements ruraux au sud de Caen à partir du III siècle avant J.-C. (fig. 11). Cette densité est d'autant plus remarquable que les différences de statut entre sites supposés contemporains semblent peu accentuées. Chaque site étudié a livré des objets métalliques, quelques amphores vinaires et du mobilier d'importation. Le dépôt intentionnel de carcasses animales et d'objets singuliers est une pratique courante comme le suggèrent les ensembles mis au jour à Fleury-sur-Orne (San Juan et al., 1994), Ifs (Le Goff et al., 2007) et Mondeville (Nillesse, 2006). Localement, il parait donc possible de remettre en cause l'existence d'une hiérarchisation marquée des établissements laténiens.

En tenant compte de ces remarques, leur répartition s'apparente à un semis régulier d'enclos domestiques. Tout se passe comme si un module se répétait à intervalles constants d'est en ouest. Un tel schéma suggère l'existence d'une organisation très resserrée du plateau. À l'intérieur de chacun des trois secteurs de Fleury-sur-Orne, Ifs et Mondeville, les distances obtenues par la méthode des polygones de Thiessen sont en effet toutes inférieures au kilomètre : les sites polariseraient alors des territoires bien plus contractés que ceux décrits par les géographes (Dunn, 1954; Chisholm, 1962).

La portée maximale des activités agricoles ne répond donc pas rigoureusement aux modèles traditionnellement admis. La taille réduite des domaines interpelle, d'autant que les contraintes topographiques sont inexistantes. Pour le moment, nous n'en sommes qu'au stade des hypothèses, mais les spécificités du système agraire bas-normand sont désormais bien documentées et autorisent l'élaboration de scénarios explicatifs stimulants.

La place prépondérante occupée par l'élevage bovin et caprin est attestée par un faisceau d'indices concordants, qu'il s'agisse des restes fauniques eux-mêmes ou de quelques structures particulières assimilables à des corrals (cf. supra). Cette activité requiert l'ouverture d'espaces suffisamment vastes pour supporter des troupeaux importants étant donné que la capacité de charge d'une prairie naturelle varie, selon les auteurs, de 0,7 à 1 équivalent bovin par hectare (Bakels, 1982; Malrain et al., 2002, p. 150). Mais ces troupeaux demeurent relativement mobiles. Il est donc probable que les parcelles enherbées, ne demandant que peu d'investissements fonciers et techniques, aient été préférentiellement implantées dans des secteurs marginaux et/ou peu favorables à la céréaliculture. Rappelons également qu'en période de montaison, les friches et les espaces boisés sont susceptibles de fournir des fourrages verts dont les propriétés nutritives sont loin d'être négligeables. Ce fait a été souligné au sujet de la basse vallée de la Dives sur la base des enregistrements polliniques recueillis. Ceux-ci témoignent d'une exploitation anthropique du couvert arbustif des coteaux de la rive gauche, limitrophe de la Plaine de Caen, tout au long de la Protohistoire et particulièrement au second âge du Fer (Carpentier, 2007). Il y aurait ainsi lieu d'étendre la surface exploitée par ces communautés gauloises de la plaine caennaise jusqu'aux pentes des vallées, dans un contexte de type outfield élargi où les surfaces boisées constituent d'appréciables réserves vivrières pour le bétail.

Le recours à l'affourragement hivernal n'influe pas de la même manière sur l'organisation spatiale des aires d'approvisionnement. Le maintien de prés de fauche nécessite des efforts considérables qui vont de la mise en défens des lopins exploités jusqu'au stockage des meules. À ces contraintes multiples s'ajoute l'éventualité d'un transport des foins vers les fermes au moyen de chariots ou de bâts. Autant d'éléments qui nous incitent à localiser ces prairies de fauche au plus près des exploitations, dans des secteurs bien desservis. Ce n'est que sensiblement plus tard en effet, dans les premiers siècles du Moyen Âge, que les systèmes prairiaux affichent leur plus grand développement dans les couloirs des vallées voisines (Carpentier, 2007).

Le tableau précédent suscite logiquement quelques commentaires. En dépit de leur taille réduite, les territoires agricoles ne semblent pas soumis à un impact anthropo-zoogène uniforme. Peut-être est-il plus satisfaisant de concevoir une organisation interne plus complexe - et sans doute moins monolithique - des domaines ruraux gaulois. Nous référant à l'exemple des systèmes à infield/outfield, nous postulons l'existence d'un gradient d'intensité de valorisation des terres agricoles au sein des territoires délimités. Ce modèle mis au point par les géographes permet d'opposer une couronne périphérique exploitée de manière plus ou moins ténue à une zone centrale cultivée en permanence et faisant l'objet d'une attention accrue de la part des paysans (Brunet et al., 1993). C'est notamment là que seraient récoltés les foins. C'est aussi là que se trouvent les parcelles emblavées et fumées dont les produits sont plus spécialement destinés à l'alimentation humaine.

La délimitation des zones d'infield repose sur le tracé d'un cercle théorique de $400 \mathrm{~m}$ de rayon autour de chaque établissement gaulois. Ce seuil a été fixé à partir de l'observation des parcellaires environnant les deux fermes d'Ifs «Object'Ifs Sud ». Sur ce gisement, les fossés bordiers ne peuvent plus être suivis ou s'estompent au-delà de ce seuil de $400-450 \mathrm{~m}$; il est évident que les processus taphonomiques doivent être pris en compte pour expliquer cet état de fait, mais il est probable que le degré d'artificialisation des milieux ait été plus faible en dehors de ce cercle, provoquant la dégradation accélérée de ces structures. Cette proposition, arbitraire au premier abord, est aussi confortée par un certain 


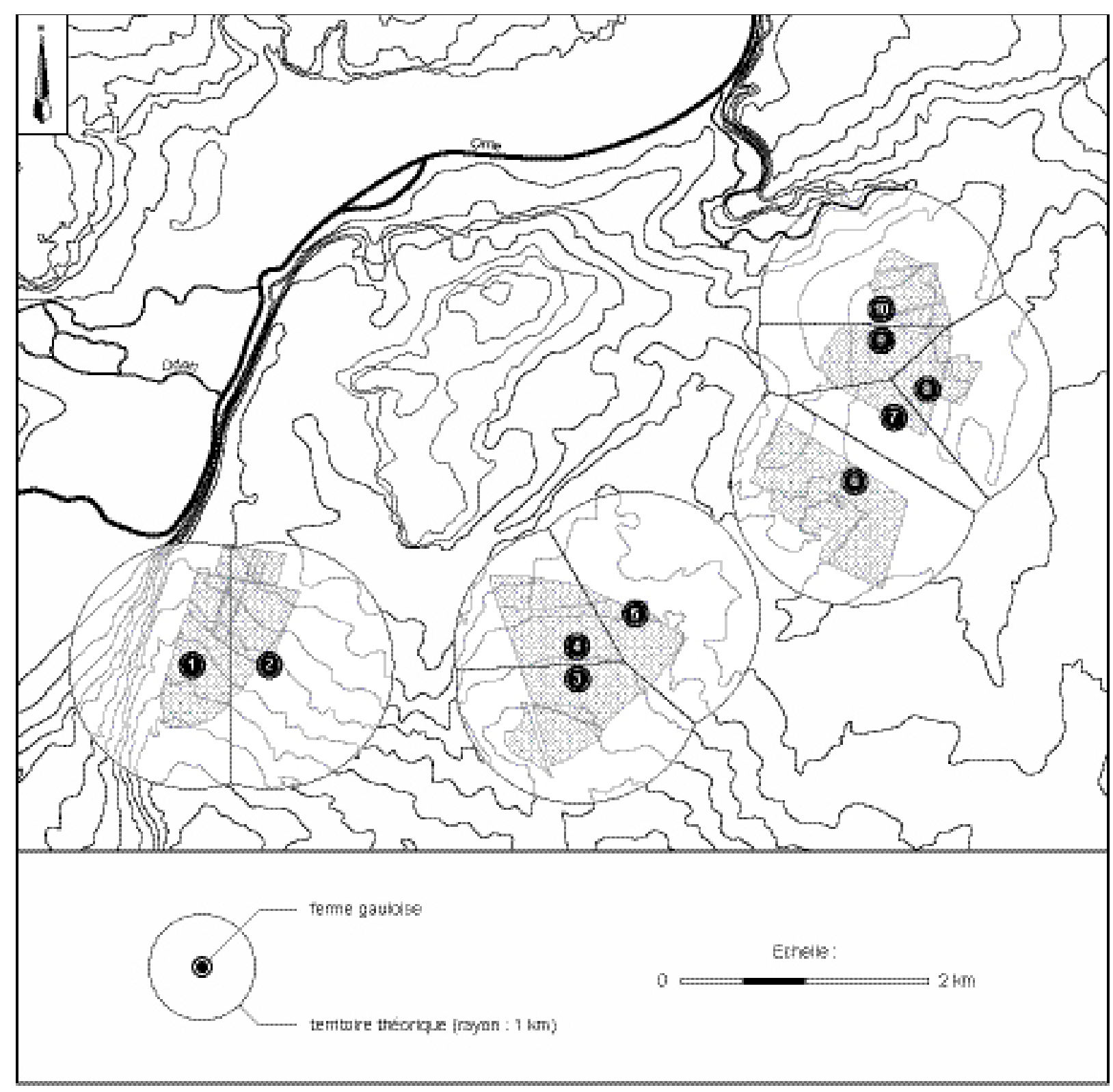

Figure 11 : Répartition des établissements agricoles gaulois du plateau de Mondeville. 1 - Fleury-sur-Orne "Parc d'Activité »; 2 - Fleury-sur-Orne "CD 120 »; 3 - Ifs "Object'Ifs Sud, ferme n 6 "; 4 : Ifs "Object'Ifs Sud, ferme n 5 »; 5 - Cormellesle-Royal «Échangeur »; 6- Mondeville " Haut-Saint-Martin»; 7 - Mondeville « l'Étoile 1 »; 8 - Mondeville « l'Étoile 2 »; 9 - Mondeville "l'Étoile 3 »; 10 - Mondeville "MIR ».

Figure 11: Location map of the Gallic farmsteads located on the Mondeville plateau.

nombre d'exemples historiques et ethnographiques auxquels les archéologues peuvent se référer (Nissen-Jaubert, 1998, p. 558; Nuninger, 2002, p. 171).

La modélisation réalisée sur ces bases montre la grande homogénéité des superficies concernées par une activité agricole soutenue (fig. 12). Les zones d'infield s'étendent en effet sur des secteurs couvrant de 30 à 45 ha environ, la valeur moyenne de 38 ha étant sans doute légèrement suré- valuée en raison de biais inhérents à l'échantillon. Entre Ifs et Fleury-sur-Orne par exemple, les vides archéologiques n'en sont peut-être tout simplement pas (des diagnostics et des fouilles sont d'ailleurs prévus dans ce secteur très prochainement). L'absence de ferme dont l'emprise territoriale pourrait concurrencer celle des domaines d' "Object'Ifs Sud » et $\mathrm{du}$ " CD 120 » est certainement à mettre sur le compte du mitage urbain qui a affecté très tôt cette portion de l'agglo- 


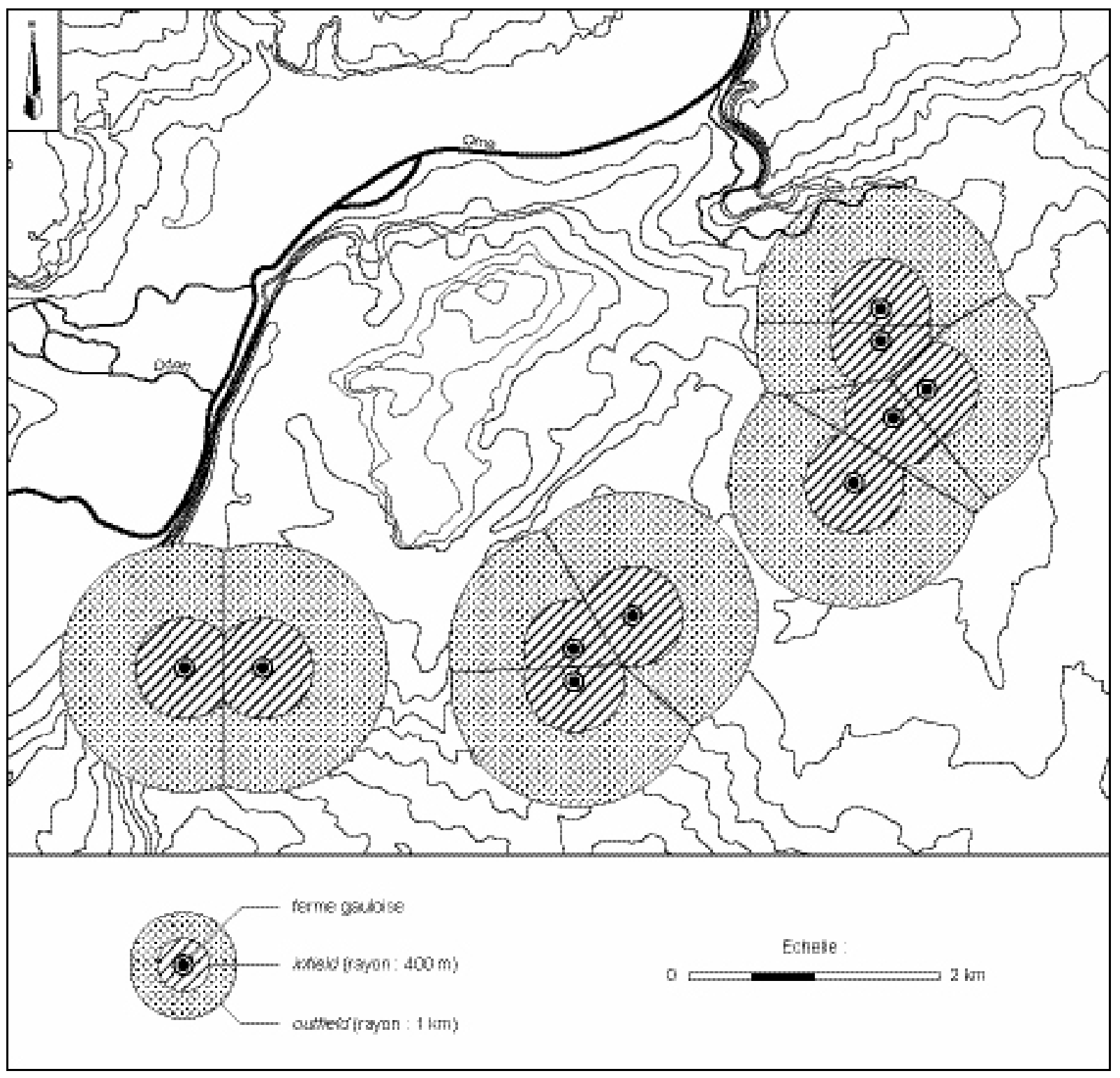

Figure 12 : Délimitation des zones d'infield et d'outfield (modélisation théorique) autour des établissements agricoles gaulois du plateau de Mondeville.

Figure 12: Demarcation of infield and outfield areas surrounding the Gallic farmsteads on Mondeville Plateau.

mération caennaise. Il en résulte une vision optimiste de l'extension globale des finages valorisés par ces deux établissements.

Quoi qu'il en soit, la surface supposée de ces zones d'infield demeure particulièrement limitée, ce qui pourrait sembler paradoxal étant donné la prospérité économique des sites dont il est question. Le constat est d'autant plus recevable que l'infield n'est jamais intégralement cultivé. Dans l'hypothèse d'un système cultural respectant le principe de la rotation biennale (Mazoyer et Roudart, 1997), seule une partie des champs est emblavée, l'autre étant laissée en jachère. Il faut donc considérer que seuls 15 à 20 ha étaient effectivement exploités pour la production de céréales, de légumineuses et éventuellement de foin; ce qui n'exclut pas le développement de cultures moins exigeantes dans l'outfield. Au-delà de ces considérations, c'est donc le problème de la viabilité des exploitations qui est posé. Cette contradiction apparente est en réalité résolue par la réintégration de données essentielles dans le modèle.

Il convient d'abord de relativiser les remarques précédentes. Les chiffres évoqués correspondent peu ou prou aux estimations obtenues par d'autres méthodes pour le 
deuxième âge du Fer (Gransar, 2001; Malrain et al., 2002, p. 150), voire pour des périodes sensiblement plus récentes (Ouzoulias, 2006). Les témoignages laissés par l'administration romaine montrent notamment que la quantité de terres que peut travailler un attelage en une année oscille entre 10 et 15 ha (Ferdière, 1988; Chouquer et Favory, 1991). En ce sens, notre démarche est en partie validée : les hypothèses de travail formulées jusqu'à présent s'inscrivent dans des fourchettes acceptables, compatibles avec d'autres évaluations.

Ceci étant établi, il est possible d'approfondir le raisonnement en examinant la répartition des différents types de substrats à l'intérieur des limites spatiales précédemment définies (fig. 13). La représentation des principales classes pédologiques composant l'infield a d'abord été calculée. Les
Figure 13 : Cartographie des entités pédologiques reconnues dans un rayon de $1 \mathrm{~km}$ autour des établissements agricoles gaulois du plateau de Mondeville.

Figure 13: Map of the main pedological units recognized within $1 \mathrm{~km}$ around the major Gallic farmsteads of the Mondeville plateau.

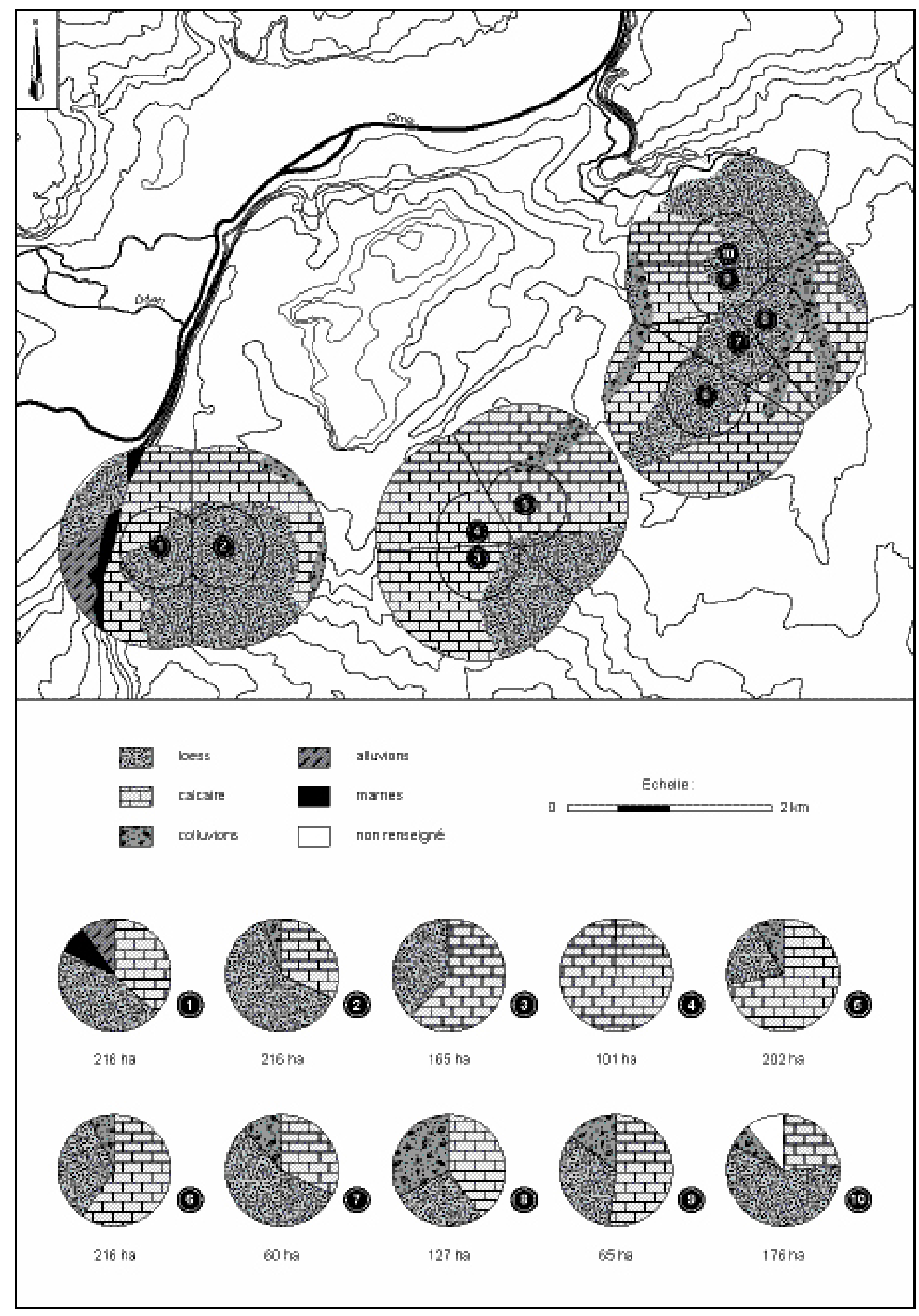


résultats ont ensuite été comparés avec la représentation relative de ces mêmes classes au sein d'un espace circulaire de $1 \mathrm{~km}$ de rayon (fig. 14). Dans le cas des sites de Mondeville et de Fleury-sur-Orne, il apparait clairement que les placages loessiques ont constitué un élément décisif dans les choix qui ont présidé à l'implantation des établissements agricoles gaulois et des parcelles cultivées attenantes. Leur surreprésentation systématique traduit l'attrait qu'ont exercé ces terres au potentiel agronomique remarquable. Le déficit de terrains lourds soumis à des contraintes hydriques plus ou moins fortes dans la zone d'infield mérite également d'être relevé. Ces deux faits vont dans le sens d'une probable spécialisation de l'infield dans les cultures végétales, les troupeaux étant préférentiellement menés vers des espaces périphériques ou parqués sur les jachères. De la même manière, dans le secteur d'Ifs, les terrains calcaires naturellement bien drainés semblent eux aussi particulièrement attractifs.

Cette prédominance systématique des terres les plus riches au sein de l'infield n'est pas surprenante. Les fermes se sont implantées dans les secteurs les plus favorables au développement d'une stratégie d'intensification agricole. Cette dernière ne se traduit pas par un accroissement des surfaces labourables mais par des changements significatifs dans les modalités d'exploitation des ressources végétales et animales.

Les analyses carpologiques réalisées par V. Matterne (Matterne, 2001, p. 105; Lepetz et Matterne, 2003; Marcigny et al., 2004) ont effectivement mis en évidence une spécialisation des établissements gaulois de la Plaine de Caen dans la culture des légumineuses (fig. 15). Ces cultures sont réputées pour les soins particuliers qu'elles réclament tout au long de leur période de croissance. En accordant une place inhabituelle à des espèces telles que le pois et la féverole, les occupants du plateau de Mondeville ont délibérément choisi de privilégier un système cultural intensif dont la pérennité dépend étroitement de l'ampleur des investissements techniques et énergétiques consentis (temps de travail et main-d'œuvre). Dans ce contexte, il a sans doute été jugé préférable de réduire la taille des exploitations et d'adapter les structures foncières aux spécificités d'un système agraire contrastant avec les systèmes extensifs du nord de la France. La configuration spatiale repérée au sud de Caen permet effectivement de gagner du temps en limitant les contraintes de déplacement et d'éviter de probables surcoûts de production. Elle n'entrave toutefois pas l'obtention d'un volume suffisant de céréales pour répondre aux besoins journaliers des exploitants dans la mesure où les terres aux potentialités les plus larges ont été systématiquement favorisées.

À court terme, les rendements obtenus avec les légumineuses sont inférieurs à ceux des céréales (Matterne, 2001). Considéré dans une perspective plus large, ce mode de faire- valoir présente toutefois plusieurs avantages. D’une part, il limite l'épuisement prématuré des sols, les légumineuses ayant la propriété de fixer l'azote atmosphérique; elles agissent donc comme des agents fertilisants, restituant au sol des quantités importantes d'azote (Gregg, 1988, p. 75; Mazoyer et Roudart, 1997). Ces qualités peuvent être mises à profit pour augmenter les rendements des champs consacrés à la céréaliculture, mais aussi des parcelles enherbées. Dans ce dernier cas, l'apport de composés azotés autorise effectivement un nombre moyen de coupes annuelles toujours supérieur à celui des prairies non fertilisées, quel que soit le mode de récolte - pâture par les animaux et/ou fauche pour produire du fourrage. Ainsi, une première coupe de printemps pour le foin peut-elle être suivie d'une ou plusieurs autres. Ce n'est que lorsque la prairie produit moins, à l'automne, qu'elle est pâturée directement par les animaux. Dans un système dépendant fortement de la disponibilité en fourrages pour l'alimentation du bétail, de telles qualités ne pouvaient être ignorées des cultivateurs.

D'autre part, les légumineuses peuvent être au moins partiellement utilisées en tant que plantes fourragères d'appoint. Leur présence renforce donc l'image d'une région vouée à l'élevage. Naturellement, la majorité des animaux est entretenue pour la production carnée et la préparation de salaisons. Si l'exploitation bouchère reste effectivement prioritaire, elle n'est toutefois pas exclusive tant il est vrai que l'accumulation de quantités substantielles de fumier devient un élément fondamental de l'agrosystème local. Mieux nourris, les animaux fournissent aussi davantage de viande et de déjections. Ces dernières contribuent à amender les terres cultivées et à augmenter les rendements. Ce fonctionnement vertueux mérite tout de même d'être relativisé dans la mesure où les procédés de transfert de fertilité, encore rudimentaires, génèrent inévitablement des pertes. L’emploi du fumier devait donc être réservé à quelques arpents soigneusement sélectionnés, contraignant les paysans à user d'autres artifices.

Enfin, les légumineuses sont des espèces semées au printemps et caractérisées par un cycle végétatif court. Cultivées dans le cadre d'une rotation biennale, elles permettent de ménager une période de jachère de près de vingt mois (Mazoyer et Roudart, 1997). Durant ce laps de temps, le sol reconstitue ses réserves en nutriments et retrouve des capacités productrices optimales, limitant ainsi le recours aux amendements organiques et minéraux.

Au-delà, c'est donc bien la recherche d'un accroissement de la productivité qui semble avoir contribué à façonner la campagne gauloise aux portes de Caen. L'espace agraire apparaît subdivisé et structuré en fonction d'impératifs à la fois techniques et économiques. Ce processus a été rendu possible non seulement par le renouvellement partiel des 

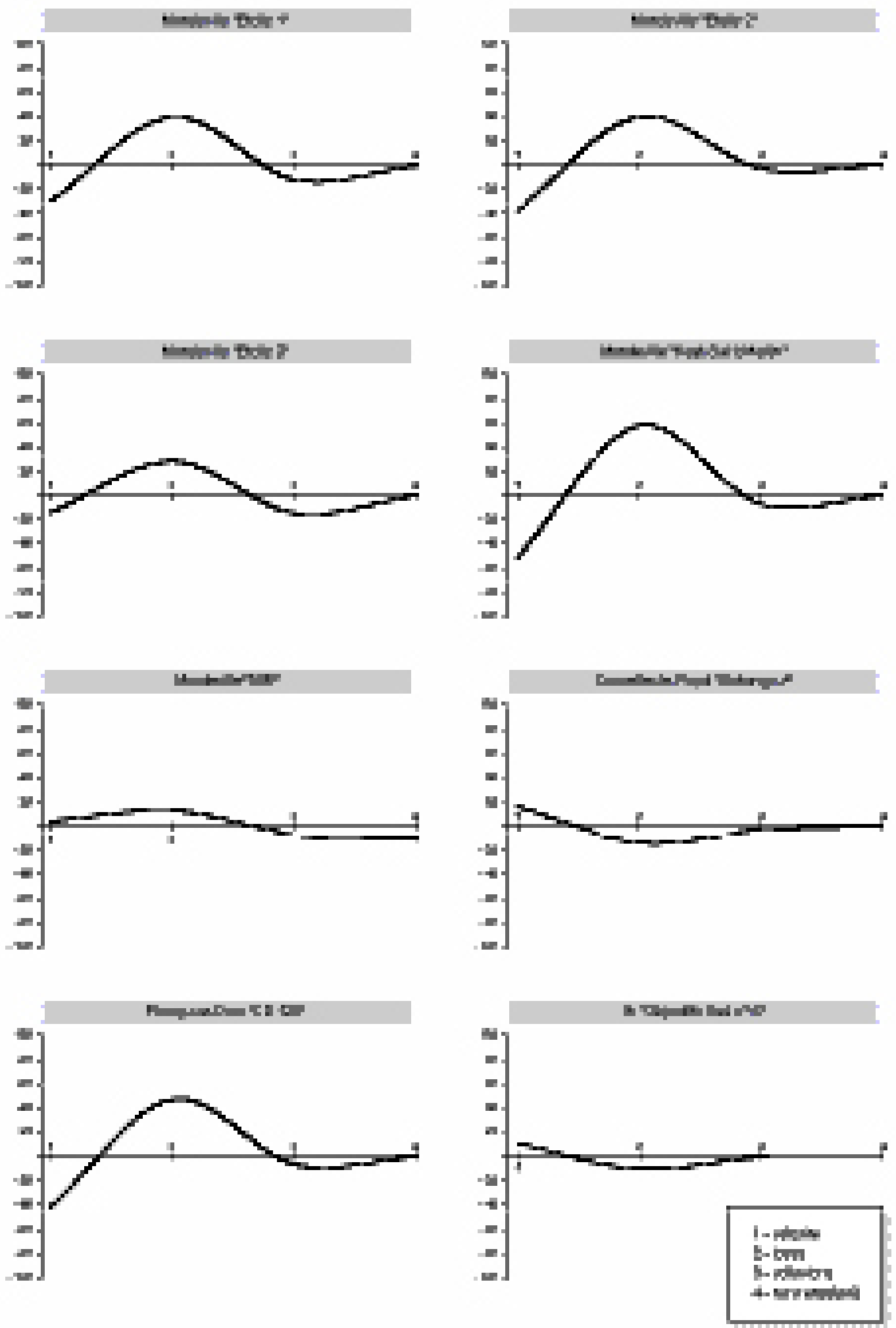

Figure 14 : Différence entre la représentation des substrats de l'infield et ceux de la zone plus distante (dans un rayon de $1 \mathrm{~km}$ ) autour des établissements agricoles gaulois du plateau de Mondeville (exprimée en pourcentages).

Figure 14: Difference between the ratio of pedological units of the infield and those of the area beyondof $1 \mathrm{~km}$ radius around the Gallic farmsteads of Mondeville plateau (stated in percentages). 


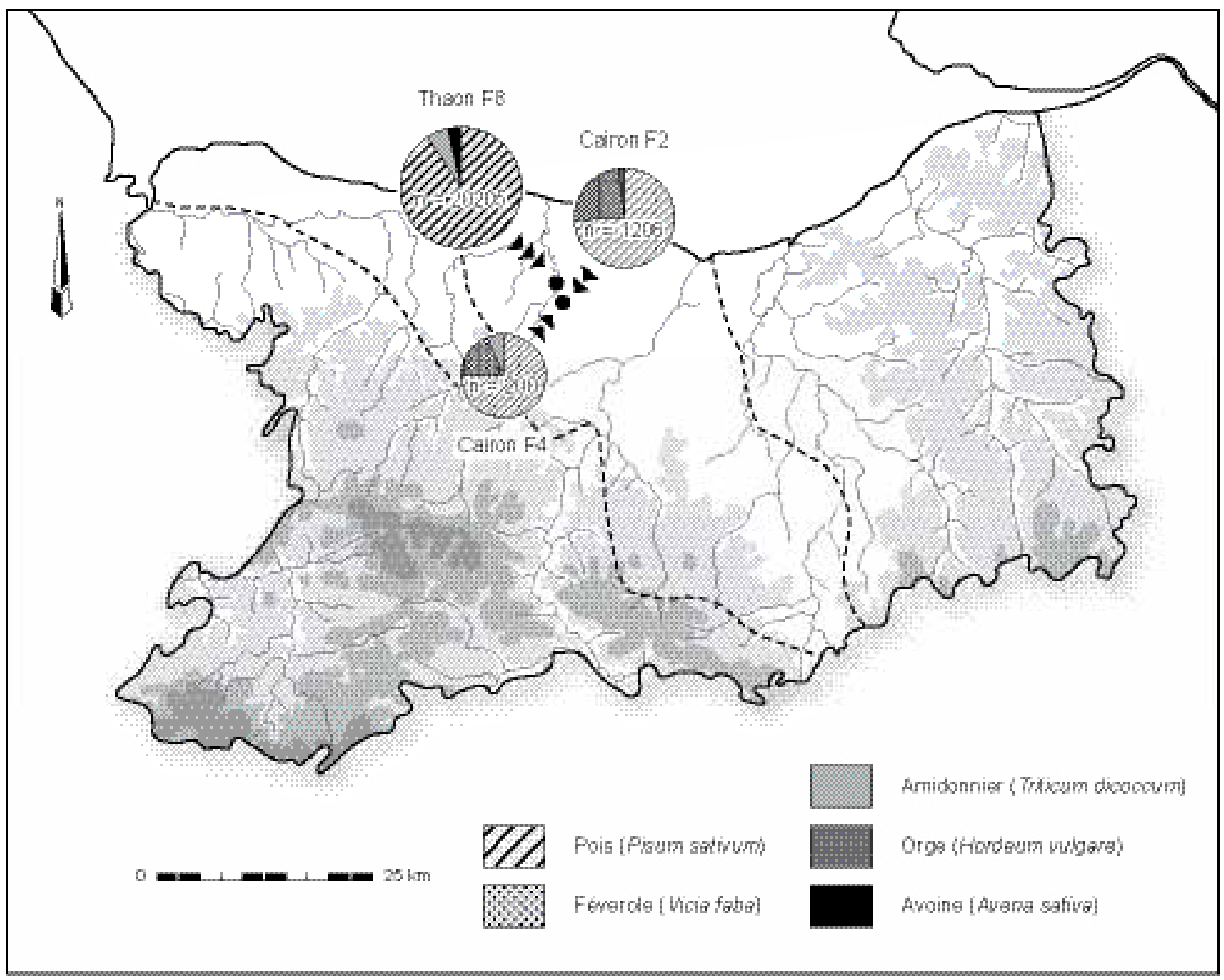

Figure 15 : Fréquence des principales espèces cultivées (légumineuses et céréales) dans la Plaine de Caen à la fin de la période gauloise, d'après le contenu de silos des $\mathrm{II}^{\mathrm{e}}$ et $\mathrm{I}^{\mathrm{er}}$ siècles av. J.-C. La surface des graphes est proportionnelle à la taille des échantillons et exprimée en échelles logarithmiques. Les données chiffrées sont issues des travaux de V. Matterne (d'après San Juan et al., 1999). A Mondeville "l'Étoile ", les pois et féveroles sont aussi très fortement représentés.

Figure 15: Frequency of archaeobotanical remains of cultivated plants (legumes and cereals) from 2nd and 1st centuries BC storage pits in the Plaine de Caen. Graph surfaces are proportional to sample sizes (in logarithmic scale), according to V. Matterne. At Mondeville "l'Etoile", peas and feverolles are also strongly represented.

façons culturales, mais aussi par la consolidation des complémentarités entre cultures et élevage. Manifestement, ces changements structurels s'enracinent bien avant la conquête romaine. $\mathrm{La}$ " romanisation» de ces populations se traduit surtout en définitive par l'adoption progressive, au cours des $\mathrm{I}^{\text {er }}$ siècles avant et après J.-C., de nouveaux matériaux de construction dont l'emploi ne se généralise véritablement dans les campagnes qu'à compter du $\mathrm{II}^{\mathrm{e}}$ siècle de notre ère. Jusqu'à cette époque, le corpus régional des sites antiques recense toutefois plusieurs exemples de petites fermes galloromaines (à Ifs, Eterville ou Caen) dont les caractères for- mels, bien qu'acquis à la mode romaine (fondations en dur, couvertures en tuiles, rectitude géométrique des enclos et systèmes de fossés...), prolongent le modèle standardisé de la "ferme indigène ", parachevé à l'époque laténienne.

Par ailleurs, nombre de villae fondées entre la seconde moitié du $\mathrm{I}^{\text {er }}$ siècle avant et la première moitié du $\mathrm{I}^{\text {er }}$ siècle après J.-C. prennent le relais, directement ou à quelques dizaines de mètres de distance seulement, d'anciens établissements gaulois dont le statut économique apparaît déjà relativement élevé (Chanson et al., 2002), et l'empreinte écologique avérée comme à Touffréville (Ponel et al., 2000; 
Guitton et Coulthard, 2007). Les exploitations agricoles du Haut-Empire s'insèrent dans un paysage déjà largement colonisé et transformé par l'homme depuis au moins le premier âge du Fer, se surimposant à d'anciennes organisations spatiales ainsi qu'aux réseaux de chemins associés aux parcellaires protohistoriques dont, selon les cas, ils épousent ou modifient le dessin et l'orientation. Ces " petits mondes ", qui ne sont encore que très localement révélés par les grands décapages archéologiques, évolueront désormais dans un contexte d'échanges dynamique, initié depuis la fin de la période gauloise et que traduisent les nombreux équipements et témoins d'importation recueillis au sein des habitats. La mutation globale qui accompagne les premiers siècles de notre ère réside en fait bien davantage dans le développement spectaculaire et parallèle des villes, marchés et voies de communication (maritimes, fluviales et terrestres), ces dernières dessinant par ailleurs les lignes de force des emprises territoriales et systèmes parcellaires de l'Antiquité romaine, dont l'empreinte durable se manifeste dans le paysage jusqu'à nos jours.

Reste à identifier les différents stimuli qui ont catalysé ces ajustements particuliers vers la fin de l'âge du Fer. Localement, la qualité des sols a pu susciter l'essor d'un système agraire performant, générant une relative prospérité, mais nous excluons d'emblée toute explication monolithique forcément simplificatrice. Il est d'ailleurs évident que la période de croissance qui s'ouvre dans le courant du III ${ }^{\mathrm{e}}$ siècle avant J.-C. est aussi conditionnée par des mutations plus profondes qui affectent en arrière-plan les structures sociopolitiques.

\section{Conclusion}

Les premiers modèles d'occupation proposés ici sont le résultat d'une politique archéologique cohérente menée depuis une quinzaine d'années par les services de l'État et conduite principalement, sur le terrain, par un seul opérateur : l'INRAP. Ce suivi des aménagements périurbains caennais (zones industrielles, lotissements), réalisé avec un souci constant de préservation du patrimoine et des archives du sol, nous a permis d'observer non seulement les sites archéologiques et leur environnement immédiat, toutes périodes confondues, mais aussi, grâce à des décapages mécaniques importants et à la réalisation de sondages systématiques, les zones considérées comme " hors sites ", regroupant réseaux parcellaires, chemins et zones dénuées de vestiges. Chaque ensemble archéologique peut ainsi être rattaché à un réseau plus large à la fonction sociale évidente. Cette accumulation de données issues de sites structurés emblématiques et de vestiges plus modestes, dont il est fait peu de cas lors des arbitrages administratifs, contribue non seulement à la reconnaissance du tissu des occupations humaines, à son évolution sur le temps long, mais aussi à l'identification des principales transformations qui affectent les différentes composantes des systèmes de production et des groupes sociaux.

Encore plus riche d'enseignements, la lecture des données dans la longue durée nous permet effectivement de dépasser le cadre étriqué de l'histoire événementielle pour nous rapprocher d'une autre notion de l'histoire, chère à Fernand Braudel : une histoire des mouvements plus lents et plus profonds qui forgent les sociétés.

Cette approche multiscalaire des données archéologiques, projetée à la fois dans le temps et dans l'espace, nous oblige à dépasser les frontières de la périodisation traditionnelle et à repenser en partie nos outils méthodologiques. Elle invite parallèlement à concevoir de nouveaux programmes d'investigation dimensionnés dès l'origine à la focale la mieux adaptée à l'observation des dynamiques sociales protohistoriques. Dans cette optique, l'approche surfacique extensive ne devrait pas susciter de réticences de la part des décideurs, d'autant que les outils dont nous disposons sont désormais à la hauteur des enjeux scientifiques. Si l'utilisation des systèmes d'information géographique permet, aujourd'hui, de mieux digérer les données et de mieux décrypter, sur de grands espaces, l'organisation puis l'évolution des sociétés qui nous ont précédés, la coordination de la recherche sur des espaces cohérents, véritables zones échantillons où il faudrait enfin concentrer les énergies, fait encore trop souvent défaut.

\section{Bibliographie}

Amiet, Y., Boutruche, M., Julien, J.-L. et Bunel, F., 1996 - Guide agronomique des sols de Basse-Normandie, Caen, Chambre régionale d'agriculture de Normandie, $111 \mathrm{p}$.

BAKELS, C. C., 1982 - Zum wirtschaftlichen Nutzungsraum einer Bandkeramischen Siedlung, in PAvúk, J. (dir.), Siedlungen der Kultur mit Linearkeramik in Europa (Internationales Kolloquium, Nové Vozokany 1981), Nitra, Archäologisches Institut der Slowakischen Akademie der Wissenschaften, p. 916.

BaKels, C.C., 1997 - The beginnings of manuring in Western Europe, Antiquity, 71, p. 272, 442-445.

Barker, G., 1999 - Cattle-keeping in ancient Europe : to live together or apart? in FABECH, C. et Ringtved, J. (dir.), Settlement and Landscape. (Proceedings of a conference in Arhus, Denmark, 1998), Århus, Jutland Archaeological Society, p. 273-280. 
Bantelmann, A., 1975 - Die frühgeschichtliche Marschensiedlung beim Elisenhof in Eiderstedt. Frankfurt, Landschaftsgeschichte und Baubefunde (Studien zur Küstenarchäologie SchleswigHolsteins, serie A, Elisenhof band 1), 190 p.

Behre, K.E. et Kucan, D., 1994 - Die Geschichte der Kulturlandschaft und des Ackerbaus in der Siedlungskammer Flögeln, Niedersachsen, seit der Jungsteinzeit, Probleme der Küstenforschung im südlichen Nordseegebiet, 21, p. 1-227.

Besnard-Vauterin, C.-C. et Navarre, N., 2008 - Une ferme de La Tène finale à l'époque gallo-romaine sur la ZAC de Beaulieu à Caen (Calvados), Revue archéologique de l'Ouest, 25, p. 163-186.

Besnard-Vauterin, C.-C., Chancerel, A. et Marcigny, C., 2006 - L'enceinte de l'âge du Bronze moyen de l'Étoile (Mondeville), in Chancerel, A., Marcigny, C. et Ghesquière, E. (dir.), Le plateau de Mondeville (Calvados) du Néolithique à l'âge du Bronze, Paris, Maison des Sciences de l'Homme (coll. "Documents d'Archéologie française ", 99), p. 111-138.

BLOCH, M., 1968 - Les caractères originaux de l'histoire rurale française (tome premier), Paris, Armand Colin, $266 \mathrm{p}$.

Bryumont, F. (dir.), 2008 - Prés et pâtures en Europe occidentale. (Actes des XXVIII journées internationales d'Histoire de l'abbaye de Flaran, septembre 2006), Toulouse, Presses universitaires du Mirail, 292 p.

Brun, P., Marcigny, C. et Vanmoerkerke, J., 2006 - Essai d'évaluation des opérations de grande surface, in BRUN, P., Marcigny, C. et Vanmoerkerke, J. (dir.), Une archéologie des réseaux locaux. Quelles surfaces étudier pour quelle représentativité? (Actes de la table ronde de Châlons-en-Champagne, juin 2005). Les Nouvelles de l'Archéologie, 104-105, p. 88-96.

Brunet, R., Ferras, R. et Théry, H., 1993 - Les mots de la Géographie. Dictionnaire critique (coll. Dynamiques du territoire), Paris, La Documentation Française/Montpellier, éd. Reclus, $518 \mathrm{p}$.

Burnouf, J., 2007 - Des milieux et des hommes : méthodes d'étude en archéologies environnementales, in Demoule, J.-P. (dir.), L'archéologie préventive dans le monde,Paris, éd. La Découverte (coll. "Recherches »), p. 117-130.

Carpentier, V., 2007 - "Les Pieds dans l'Eau... ». La basse Dives et ses riverains, des origines aux temps modernes. Contribution à l'histoire environnementale des zones humides et littorales de Normandie (thèse de doctorat), Caen, Université de Caen Basse-Normandie (7 vol.), 2186 p.

Carpentier, V., Ghesquière, E. et Marcigny, C., 2006 - Grains de sel. Sel et salines du littoral bas-normand (Préhistoire-XIX siècle). Entre Archéologie et Histoire, Rennes, coédition CeRAAAMARAI, $182 \mathrm{p}$.

Carpentier, V., Garnier, E., Lespez, L. et Maertens, S., 2007a - Les marais de la basse vallée de la Dives. Contribution interdisciplinaire à l'histoire d'un espace productif et de ses mutations paysagères sur le temps long, in BeCK, C., Benarrous,
R., Derex, J.-M. et Gallicié, A. (dir.), Les zones humides européennes: espaces productifs d'hier et d'aujourd'hui. (Actes du $I^{e r}$ colloque international du Groupe d'Histoire des Zones humides, Le Blanc 2005). Astuaria, 9, p. 213-230.

Carpentier, V, Ghesquière, E. et Marcigny, C., 2007b Archéologie en Normandie. Rennes, éd. Ouest-France, 128 p.

Chancerel, A., Marcigny, C. et Ghesquière, E. (dir.), 2006a - Le plateau de Mondeville (Calvados) du Néolithique à l'âge du Bronze, Paris, Maison des Sciences de l'Homme (coll. "Documents d'Archéologie française ", 99), 205 p.

Chancerel, A., Marcigny, C. et San Juan, G., 2006b - La double enceinte de l'âge du Bronze moyen de la ZI sud (Mondeville, Grentheville), in Chancerel, A., Marcigny, C. et Ghesquière, E. (dir.), Le plateau de Mondeville (Calvados), du Néolithique à l'âge du Bronze, Paris, Maison des Sciences de l'Homme (coll. "Documents d'Archéologie française », 99), p. 140-172.

Chanson, K., Le Gaillard, L. et Paez-Rezende, L., 2002 Images de la romanisation d'un établissement agricole de la plaine de Caen entre les $\mathrm{IV}^{\mathrm{e}}$-III ${ }^{\mathrm{e}}$ siècles avant et le $\mathrm{III}^{\mathrm{e}}$ siècle après J.-C. : Fleury-sur-Orne, Archéopages, 6, p. 4-9.

Chisholm, M., 1962 - Rural settlement and land use: an essay in location, London, Hutchinson, 207 p.

Chouquer, G. et Favory, F., 1991 - Les paysages de l'Antiquité. Terres et cadastres de l'Occident romain, Paris, Errance (coll. "Les Hespérides »), 213 p.

Clet-Pellerin, M. et Verron, G., 2004 - Influence de l'homme sur l'évolution des paysages normands pendant l'Holocène, in Richard, H. (dir.), Néolithisation précoce. Premières traces d'anthropisation du couvert végétal à partir des données polliniques. Besançon, Presses universitaires Franc-comtoises (coll. "Annales Littéraires ", 777; "Environnement, sociétés et archéologie ", 7), p. 53-68.

Columelle - De l'Agriculture. (Texte établi, traduit et commenté par Dumont, J.-C.), Paris, Les Belles Lettres.

Coste, N., Guilaine, J. et Revel, J.-C., 1988 - Archéologie et pédologie : essai de reconnaissance des territoires d'exploitation autour des sites néolithiques, Bulletin de la Société préhistorique française, 85 (10-12), p. 390-411.

Diomard, I., Chégard, L. et LeVeau, J.-M., 2006 - Synthèse des résultats d'analyses de terre. Basse-Normandie 1999-2005, SaintLô, Laboratoire Agronomique de Normandie, 29 p.

DunN, E. S., 1954 - The location of agricultural production, Gainesville, University of Florida Press, 115 p.

Ferdière, A., 1988 - Les campagnes en Gaule romaine (tome 1). Les hommes et l'environnement en Gaule rurale (52 av. J.-C.-486 apr. J.-C.), Paris, Errance, 302 p.

Ferdière, A., 1991 - Gaulois et Gallo-romains : techniques et outillages agricoles, in GuILAIne, J. (dir.), Pour une archéologie agraire : à la croisée des sciences de l'homme et de la nature, Paris, Armand Colin, p. 81-101. 
Ferdière, A., Malrain, F., Matterne, V., Méniel, P. et NissenJAUbert, A., 2006 - Histoire de l'agriculture en Gaule (500 av. J.-C.-1000 apr. J.-C.), Paris, Errance, 231 p.

FoKKens, H., 1991 - Bronze Age settlements in the Netherlands, In Chevillot, C. et Coffyn, A. (dir.) - L'âge du Bronze atlantique : ses faciès de l'Écosse à l'Andalousie et leurs relations avec le Bronze continental et la Méditerranée, Beynac, Association des Musées du Sarladais, p. 77-88.

Gransar, F., 2001 - Le stockage alimentaire à l'âge du Fer en Europe tempérée (thèse de doctorat), Paris, Université de Paris 1 (Panthéon-Sorbonne), $566 \mathrm{p}$.

Gregg, S. A., 1988 - Foragers and Farmers. Population Interaction and Agricultural Expansion in Prehistoric Europe. Chicago, The University of Chicago Press (coll. "Prehistoric Archaeology and Ecology Series »), 275 p.

GrundY, J. E., 1970 - Notes on the relationship between climate and cattle housing, Vernacular Architecture, 1, p. 3-5.

Guitton, V. et Coulthyar, N., 2007 - L'environnement forestier et l'artisanat du bois à Touffréville "La Saussaye " entre la fin du II ${ }^{\mathrm{e}}$ siècle et le IV ${ }^{\mathrm{e}}$ siècle après J.-C. (Calvados), in Querré, G. (dir.), Journée "Civilisations atlantiques et Archéosciences", Rennes 2007 (résumés des communications), Rennes, Université de Rennes 1, p. 7-10.

Heuzé, G., 1875 - La France agricole, Paris, Imprimerie nationale, $60 \mathrm{p}$.

Hitıer, H., 1903 - Le village picard, Annales de Géographie, 12, 62, p. 109-119.

Jahier, I., Besnard-Vauterin, C.-C., Lepaumier, H., Paris, P., Renault, V., San Juan, G., Dron, J.-L., Hincker, V. et Forfait, N., 2000 - Les bâtiments des habitats de l'âge du Fer en Basse-Normandie : panorama des découvertes, in MARION, S. et Blancquaert, G. (dir.), Les installations agricoles de l'âge du Fer en France septentrionale, Paris, éd. De la Rue d'Ulm/ Presses de l'ENS, p. 339-357.

Lautridou, J.-P., Auffret, J.-P., Coutard, J.-P. et Lavolle, M., 2000 - Synthèse sur les formations superficielles cénozoïques de Basse-Normandie (feuille de Caen à 1/250 000), Géologie de la France, 4, p. 57-69.

Lebece, S., 1980 - De la protohistoire au haut Moyen Âge : le paysage des terpen le long des côtes de la mer du Nord, spécialement dans l'ancienne Frise. Le paysage rural. Réalités et représentations (Actes $d u X^{e}$ congrès des Historiens médiévistes français de l'Enseignement supérieur, Villeneuve-d'Ascq 1979). Revue du Nord, 62 (244), p. 125-154.

Le Goff, E., Auxiette, G., Le Goff, I., Boulestin, B., Chérel, A.-F. et Pilet-Lemière, J., 2007 - Manifestations et pratiques cultuelles au sein des habitats et du territoire agraire laténien de la «ZAC Object'Ifs Sud » à Ifs (Calvados) : un exemple de l'ouest de la Gaule, in Daubigney, A., Barral, P., Dunning, C., Kaenel, G. et Roulière-Lambert, M.-J. (dir.), L’âge $d u$ Fer dans l'arc jurassien et ses marges. Dépôts, lieux sacrés et territorialité à l'âge du Fer (volume 2) (Actes du XXIX colloque de l'Association française pour l'étude de l'Âge du Fer, Bienne 2005), Besançon, Presses universitaires de Franche-Comté (coll. "Annales littéraires de l'université de Franche-Comté »), p. 579-584.

Lepaumier, H., San Juan, G. et Verney, A., 2006 - Les âges des Métaux, in Hincker, V. et Dron, J.-L. (dir.), 7000 ans d'histoire. Gestes funéraires de la Préhistoire à nos jours en BasseNormandie. Condé-sur-Noireau, éd. NEA, 60 p.

Lepetz, S. et Matterne, V., 2003 - Élevage et agriculture dans le nord de la Gaule durant l'époque gallo-romaine : une confrontation des données archéozoologiques et carpologiques, in Lepetz, S. et Matterne, V. (dir.), Cultivateurs, éleveurs et artisans dans les campagnes de Gaule romaine. Matières premières et produits transformés. (Actes du VIe colloque de l'association AGER, Compiègne 2002). Revue archéologique de Picardie, 1-2, p. 23-36.

Lespez, L., Clet-Pellerin, M., Davidson, R. et Marcigny, C., 2004 - Évolution des paysages et anthropisation depuis le Néolithique dans la péninsule de La Hague (Normandie), Revue d'Archéométrie, 28, p. 71-88.

Lorren, C., 1983 - De l'Antiquité au Moyen Âge, un exemple de continuité de l'habitat: le village de Saint-Martin de Mondeville (Calvados). Premiers résultats des fouilles. La Normandie. Études archéologiques. (Actes du $105^{\circ}$ Congrès national des Sociétés savantes, Caen 1980), Paris, CTHS, p. 99122.

Lorren, C., 1989 - Le village de Saint-Martin de Trainecourt à Mondeville (Calvados), de l'Antiquité au haut Moyen Âge, in Atsma, H. (dir.), La Neustrie. Les pays au nord de la Loire de 650 à 850. (Actes du XXI colloque historique franco-allemand de l'Institut historique allemand de Paris, Rouen 1985), Sigmaringen, J. Thorbecke Verlag (Beihefte der Francia, 16, 2), p. 439-466.

Malrain, F., Matterne, V. et Méniel, P., 2002 - Les paysans gaulois (IIt siècle-52 av. J.-C.), Paris, Errance/INRAP, 236 p.

MarcignY, C., 1995 - L'occupation protohistorique et galloromaine précoce, in Renault, V. (dir.) - Mondeville "HautSt-Martin". (Document final de synthèse de sauvetage urgent), Caen, Service régional de l'Archéologie de Basse-Normandie, p. 54-77.

MarCignY, C. (dir.), 2003 - Projet collectif de recherche " Habitats et occupation du territoire à l'âge du Bronze et au début du premier âge du Fer en Basse-Normandie » (Rapport d'activité 2003), Caen, Service régional de l'Archéologie de Basse-Normandiel INRAP, 158 p.

Marcigny, C., Lepaumier, H., Carpentier, V., Clément-Sauleau, S., Matterne-Zeck, V., Ghesquière, E. et Giazzon, D., 2004 - L'établissement agricole à caractère aristocratique du second âge du Fer de Saint-Martin-des-Entrées (Calvados), Revue archéologique de l'Ouest, 21, p. 63-95. 
Marcigny, C., Cément-Sauleau, S., Ghesquière, E. et Verney, A., 2005 - L'âge du Bronze en Basse-Normandie : définition par le mobilier céramique. Un premier état des lieux, in Bourgeors, J. et Talon, M. (dir.) - L'âge du Bronze du nord de la France dans son contexte européen (Actes du $125^{e}$ congrès national des Sociétés historiques et scientifiques, Lille 2000), Paris, éd. du CTHS, p. 303-332.

Marcigny, C., Flotté, D., Desloges, J. et Renault, V., 2006 - Les petits ruisseaux font les grandes rivières. L'exemple de la périphérie sud de Caen (Calvados), in Brun, P., Marcigny, C. et Vanmoerkerke, J. (dir.), Une archéologie des réseaux locaux. Quelles surfaces étudier pour quelle représentativité? (Actes de la table ronde de Châlons-en-Champagne, juin 2005). Les Nouvelles de l'Archéologie, no 104-105, p. 61-63.

Marcigny, C., Lespez, L., Ghesquière, E. et Clet-Pellerin, M., 2007 - Emprise ou déprise agricole à l'âge du Bronze moyen sur le littoral de la Manche? Une lecture du phénomène grâce aux sites normands, in Richard, H., MAGNY, M. et Mordant, C. (dir.), Environnements et cultures à l'âge du Bronze en Europe occidentale (Actes du $129^{\circ}$ Congrès national des Sociétés historiques et scientifiques, Besançon 2004), Paris, éd. du CTHS, p. 311-326.

Matterne, V., 2001 - Agriculture et alimentation végétale durant l'âge du Fer et l'époque gallo-romaine en France septentrionale. Montagnac, éd. Monique Mergoil (coll. "Archéologie des Plantes et des Animaux ", 1), 310 p.

Mazoyer, M. et Roudart, L., 1997 - Histoire des agricultures du Monde, du Néolithique à la crise contemporaine. Paris, Seuil, $533 \mathrm{p}$.

Medens, F. M. et BeAsley, M., 2001 - Roman seasonal wetland pasture exploitation near Nash, on the Gwent Levels, Wales, Britannia, 32, p. 143-184.

NiLlesse, O., 2006 - Les dépôts d'objets en fer dans les établissements ruraux gaulois de l'ouest de la France. Le rituel est-il au fond de la poubelle? in Bataille, G. et Guillaumet, J.P. (dir.), Les dépôts d'objets métalliques au second âge du Fer en Europe tempérée (Actes de la table ronde de Bibracte, octobre 2004). Glux-en-Glenne, Centre archéologique européen de Bibracte (coll. «Bibracte", 11), p. 221-246.

Nissen-Jaubert, A., 1998 - Les finages et leurs rendements : l'exemple danois, in Feller, L., Mane, P. et Piponnier, F. (dir.), Le village médiéval et son environnement. Études offertes à Jean-Marie Pesez, Paris, Publications de la Sorbonne, (coll. "Histoire ancienne et médiévale », 48), p. 551-570.

Nuninger, L., 2002 - Peuplement et territoires protohistoriques $d u$

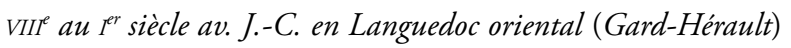
(thèse de doctorat), Besançon, Université de Franche-Comté, $290 \mathrm{p}$.

Ouzoulias, P., 2006 - L'économie agraire de la Gaule : aperçus historiographiques et perspectives archéologiques (thèse de doctorat), Besançon, Université de Franche-Comté, 250 p.
Palladius - Traité d'Agriculture (Livres I et II). (Texte établi, traduit et commenté par Martin, R.), Paris, Les Belles Lettres, $289 \mathrm{p}$.

Ponel, P., Matterne, V., Coulthard, N. et Yvinec, J.-H., 2000 - La Tene and Gallo-Roman natural Environments and human Impact at the Touffréville rural Settlement, reconstructed from Coleoptera and Plant Macroremains (Calvados, France), Journal of Archaeological Science, 27 (11), p. 1055-1072.

Poitou, C., 2007 - L'agriculture dans le canton de Patay vers 1810, Revue archéologique du Loiret, 30-31, p. 81-87.

Rasmussen, P., 1990 - Leaf-foddering in the earliest Neolithic agriculture. Evidence from Switzerland and Denmark, Acta archaeologica, 60, p. 71-86.

-, 1991 - Leaf-foddering of livestock in the Neolithic. Archaeobotanic evidence from Weier, Switzerland, Journal of Danish Archaeology, 8, p. 51-71.

-, 1993 - Analysis of Goat/Sheep Faeces from Egolzwil 3, Switzerland. Evidence for Branch and Twig Foddering of Livestock in the Neolithic, Journal of Archaeological Science, 20 (5), p. 479-502.

Rippon, S., 2000a - Exploitation and Modification. Changing Patterns in the use of Coastal Resources in Southern Britain during the Roman and Medieval periods, in ABERG, A. et Lewis, C. (dir.), The Rising Tide. Archaeology and Coastal Landscapes, Oxford, Oxbow Books, p. 65-74.

Rippon, S., 2000b - The Romano-British exploitation of coastal wetlands : survey and excavation on the North Somerset Levels, 1993-1997, Britannia, 31, p. 69-200.

RuAs, M.-P., 2008 - Prés, prairies, pâtures : éclairages archéobotaniques, in Brumont, F. (dir.), Prés et pâtures en Europe occidentale. (Actes des XXVIII journées internationales d'Histoire de l'abbaye de Flaran, septembre 2006), Toulouse, Presses universitaires du Mirail, p. 13-44.

San Juan, G., Méniel, P., Alduc-Lebagousse, A., Pilet-Lemière, J. et JAHIER, I., 1994 - Un établissement rural du $\mathrm{I}^{\mathrm{er}}$ s. avant J.-C. à Fleury-sur-Orne (Calvados), Revue archéologique de l'Ouest, 11, p. 131-164.

San Juan, G., Méniel, P., Matterne-Zeck, V., Savary, X. et JARDEL, K., 1999 - L'occupation gauloise au nord-ouest de Caen. L'évaluation du plateau de Thaon (Calvados), Revue archéologique de l'Ouest, 16, p. 131-194.

Serneels, V., 2007 - L'économie du fer protohistorique : de la production à la consommation du métal, in Milcent, P.-Y. (dir.), L'économie du fer protohistorique : de la production à la consommation du métal. (Actes du xxvin' ${ }^{\mathrm{e}}$ colloque de l'Association française pour l'Étude de l'Âge du Fer, Toulouse 2004), Aquitania, suppl. 14/2, p. 425-434.

Servais, F., 1925 - Le Vexin français, Annales de Géographie, 34 (191), p. 413-422.

Sigaut, F., 1998 - Le fer dans l'agriculture, in Feller, L., Mane, P. et Piponnier, F. (dir.), Le village médiéval et son environ- 
nement. Études offertes à Jean-Marie Pesez. Paris, Publications de la Sorbonne (coll. " Histoire ancienne et médiévale ", 48), p. 413-426.

Simons, A., 1989 - Bronze- und eisenzeitliche Besiedlung in den Rheinische Lössbörden. Archäologische Siedlungsmuster im Braunkohlengebiet, Oxford, Hadrian Books (British Archaeological Reports - International Series, 467), 291 p.

Taupin, M.-C., 1996 - Le hameau de Trainecourt, XIII ${ }^{\mathrm{e}}-\mathrm{XV}^{\mathrm{e}}$ siècle, in Fridrich, J. et KLÁpšTĚ, J. (dir.), Ruralia I. (Actes de la conférence Ruralia, Prague 1995). Památky Archeologické, suppl. 5, p. 211-216.

Thouvenot, S., Gransar, F., Auxiette, G. et Pommepuy, C., 2000 - La gestion du terroir des établissements ruraux de La Tène finale dans la vallée de l'Aisne. Un essai de modélisation : le méandre de Bucy-le-Long (Aisne), in Marion, S. et Blancquaert, G. (dir.), Les installations agricoles de l'âge du Fer en France septentrionale, Paris, éd. de la Rue d'Ulm, Presses de l'ENS, p. 157-167.

Troels-Smith, J., 1984 - Stall feeding and field manuring in Switzerland about 6000 years ago, Tools and Tillage, 5, (1), p. 13-25.

VANNiÈre, B., 2001 - Diversité des sols et potentialités agronomiques pour l'Antiquité, in Batardy, C., Buchsenschutz, O. et Dumasy, F. (dir.), Le Berry antique. Atlas 2000, Revue archéologique du Centre de la France, suppl. 21, p. 44-51.
VARron -Economie rurale (Livre II). (Texte établi, traduit et commenté par Guiraud, C., Paris, Les Belles Lettres, 172 p.

ZimmermanN, W. H., 1978 - Economy of the Roman Iron Age Settlement at Flögeln, Kr. Cuxhaven, Lower Saxony. Husbandry, Cattle Farming and Manufacturing, in Cunliffe, B. et Rowley, T. (dir.), Lowland Iron Age Communities in Europe, Oxford, Hadrian Books («British Archaeological Reports - International Series », 48), p. 147-165.

ZimmermanN, W. H., 1999a - Favourable conditions for cattle farming, one reason for the Anglo-Saxon migration over the North Sea? About the byre's evolution in the area south and east of the North Sea and England, in Sarfatij, H., Verwers, W. J. H. et Woltering, P. J. (dir.), In discussion with the past. Archaeological studies presented to W. A. Van Es. ZwolleAmersfoort, Stichting Promotie Archeologie-Rijkdienst voor het Oudheidkunding Bodemonderzoek, p. 129-144.

ZimmermanN, W. H., 1999b - Why was cattle-stalling introduced in prehistory? The significance of byre and stable and of outwintering, in Fabech, C. U. et Ringtved, J. (dir.), Settlement and Landscape. (Proceedings of a conference in Århus, Denmark, 1998), Århus, Jutland Archaeological Society, p. 295-312.

ZolA, E., 1889 - La Terre, Paris, Flammarion, 472 p. 
